\title{
ASYMPTOTIC BEHAVIOUR FOR LOCAL AND NONLOCAL EVOLUTION EQUATIONS ON METRIC GRAPHS WITH SOME EDGES OF INFINITE LENGTH
}

\author{
LIVIU I. IGNAT, JULIO D. ROSSI, AND ANGEL SAN ANTOLIN
}

\begin{abstract}
We study local (the heat equation) and nonlocal (convolution type problems with an integrable kernel) evolution problems on a metric connected finite graph in which some of the edges have infinity length. We show that the asymptotic behaviour of the solutions to both local and nonlocal problems is given by the solution of the heat equation, but on a star shaped graph in which there is only one node and as many infinite edges as in the original graph. In this way we obtain that the compact component that consists in all the vertices and all the edges of finite length can be reduced to a single point when looking at the asymptotic behaviour of the solutions. For this star shaped limit problem the asymptotic behaviour of the solutions is just given by the solution to the heat equation in a half line with a Neumann boundary condition at $x=0$ and initial datum $(2 M / N) \delta_{x=0}$ where $M$ is the total mass of the initial condition for our original problem and $N$ is the number of edges of infinite length. In addition, we show that solutions to the nonlocal problem converge, when we rescale the kernel, to solutions to the heat equation (the local problem), that is, we find a relaxation limit.
\end{abstract}

\section{INTRODUCTION}

The aim of this paper is to study solutions to diffusion equations both local and non-local in a metric graph. A metric graph is by definition a combinatorial graph where the edges, denoted by $\left\{e_{j}\right\}$ are considered as intervals of the real line $\left\{I_{j}\right\}$ with a distance on each one of them. These edges/intervals are glued together according to the combinatorial structure. We assume here that at least one of the edges is not bounded (it has infinite length).

Metric graphs have received lot of attention in recent years both from the point of view of pure mathematicians and also from potential applications. The name quantum graph is used for a graph considered as an one-dimensional singular variety and equipped with a differential operator (local or in some cases nonlocal). There are several reasons for studying quantum graphs. They naturally arise as simplified (due to reduced dimension) models in mathematics, physics, chemistry, and engineering (e.g., nanotechnology and microelectronics), when one considers propagation of waves of various nature (electromagnetic, acoustic, etc.) through a quasione-dimensional system (often a mesoscopic one) that looks like a thin neighborhood of a graph. We refer to the survey [35] and references therein.

Key words and phrases. Nonlocal diffusion, local diffusion, quantum graphs, compactness arguments, asymptotic behaviour

2020 Mathematics Subject Classification. 35B40, 45G10, 46B50. 
Let $\Gamma$ be a metric graph $\Gamma=\Gamma_{f} \cup \Gamma_{\infty}$ where $\Gamma_{f}$ is made from the finite length edges of the graph $\Gamma$ whereas $\Gamma_{\infty}$ collects all the infinite edges. First of all in this paper, we introduce $\Delta_{\Gamma}$, the Laplace operator on a metric graph $\Gamma$. Associated to the Laplacian we have an initial valued problem; the classical heat equation on $\Gamma$. This problem is well-posed and its solutions decay as time goes to infinity in a similar way as the solutions of the Cauchy problem on the whole line [25], 20], 34]. We prove that the asymptotic behaviour (in terms of the existence of an asymptotic profile) of those solutions is comparable to the solution of the classical heat equation in the half line with Neumann boundary conditions at $x=0$ and initial data at $t=0$ given by a multiple of a Dirac mass at $x=0$.

Theorem 1.1. Let $\mathbf{u}$ the solution to the heat equation in $\Gamma$ with integrable initial datum. Then, for any $1 \leq p \leq \infty$,

$$
t^{\frac{1}{2}\left(1-\frac{1}{p}\right)}\left\|\mathbf{u}(t)-\mathbf{U}_{M}(t)\right\|_{L^{p}\left(\Gamma_{\infty}\right)} \rightarrow 0, \quad \text { as } t \rightarrow \infty,
$$

and

$$
t^{\frac{1}{2}}\left\|\mathbf{u}(t)-\mathbf{U}_{M}(t)\right\|_{L^{p}\left(\Gamma_{f}\right)} \rightarrow 0, \quad \text { as } t \rightarrow \infty,
$$

where $M$ is the total mass of the initial datum and

$$
\mathbf{U}_{M}(x, t)=\frac{2 M}{N} t^{-\frac{1}{2}} \begin{cases}G(x / \sqrt{t}), & x \in \Gamma_{\infty}, \\ G(0), & x \in \Gamma_{f} .\end{cases}
$$

Here $G(s)$ is given by the classical Gaussian profile,

$$
G(s)=\frac{1}{\sqrt{4 \pi}} e^{-\frac{s^{2}}{4}} .
$$

Notice that here the classical Gaussian profile appears as $\mathbf{U}_{M}(x, t)$ is the solution to the heat equation in a half-line with Neumann boundary conditions.

Now, let us turn our attention to nonlocal diffusion equations with a convolution kernel. Equations of the form

$$
u_{t}(x, t)=(J * u-u)(x, t)=\int_{\mathbb{R}} J(x-y) u(y, t) d y-u(x, t),
$$

and variations of it, have been recently widely used to model diffusion processes. Here, and in what follows, $J: \mathbb{R} \mapsto \mathbb{R}_{+}$belongs to $L^{1}(\mathbb{R}) \cap L^{1}\left(\mathbb{R},|x|^{2}\right)$ and is assumed to be symmetric, $J(z)$ is non-increasing for $z>0$, nonnegative in $\mathbb{R}$ and strictly positive in neighbourhood of the origin. To simplify a little the statements we will also assume that $A=1$ where

$$
A:=\frac{1}{2} \int_{\mathbb{R}} z^{2} J(z) d z<\infty
$$

and comment on the general case when appropriate. Notice that, when $\varphi$ is a smooth function, a simple Taylor expansion gives

$$
\varepsilon^{-3} \int_{I} J\left(\frac{|x-y|}{\varepsilon}\right)(\varphi(y)-\varphi(x)) d y \approx\left(\frac{1}{2} \int_{\mathbb{R}} J(z) z^{2} d x\right) \varphi_{x x}(x)
$$

for $x$ an interior point of an interval $I$. As a consequence for a general $A$ the limit profiles should be rescaled correspondingly. 
When $J$ has unit integral, as stated in [21, if $u(x, t)$ is thought of as a density at the point $x$ at time $t$ and $J(x-y)$ is thought of as the probability distribution of jumping from location $y$ to location $x$, then $\int_{\mathbb{R}} J(y-x) u(y, t) d y=(J * u)(x, t)$ is the rate at which individuals are arriving at position $x$ from all other places and $-u(x, t)=-\int_{\mathbb{R}} J(y-x) u(x, t) d y$ is the rate at which they are leaving location $x$ to travel to all other sites. This consideration, in the absence of external or internal sources, leads immediately to the fact that the density $u$ satisfies equation (1.2). These kind of nonlocal equations are used to model very different applied situations, for example in biology (see [10], [36]), image processing ([32], [24]), particle systems ([7]), coagulation models ([22]), etc.

Our next goal is to study nonlocal evolution problems with a convolution kernel on the metric graph $\Gamma$, that is, we deal with

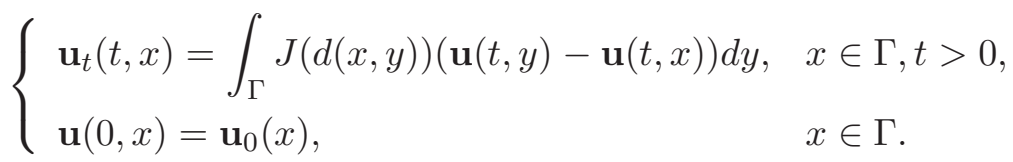

Here $d(x, y)$ is the natural distance in $\Gamma$, see Section 2. Now, particles can jump inside the graph from position $x$ to position $y$ according to the probability kernel $J(d(x, y)$ ) (an interpretation similar to the one previously given also holds here). Notice that particles may jump from one edge to another when the distance between points in the edges makes $J(d(x, y))$ positive.

For general nonlocal problems of this kind we refer to [41] that contains closely related results concerning existence and uniqueness of solutions and for different problems of this kind in the Euclidean setting to [12, 14, 16, 17, 23, 26, 30, 28] and the book [1. For the decay rate and the asymptotic profile in the real line (that coincides with the ones for the local heat equation) we refer to [14. For a class of kernels that give exponential decay we quote [31.

Here, we show that nonlocal problems are closely related to the local heat equation. In fact, one can obtain solutions to the heat equation by approximating them by solutions to nonlocal problems. To see this, for a rescaling parameter that acts in the kernel, $\varepsilon>0$, considering $J_{\varepsilon}(d(x, y))=\varepsilon^{-3} J(d(x, y) / \varepsilon)$, we deal with a relaxation limit for this nonlocal evolution problem and obtain that its solutions converge to the solutions of the local one when $\varepsilon$ goes to zero.

Theorem 1.2. It holds that the solutions to the rescaled nonlocal problem with a fixed initial datum verify

$$
\mathbf{u}^{\varepsilon} \rightarrow \mathbf{u} \text { weakly in } L^{2}\left([0, \infty), L^{2}(\Gamma)\right) .
$$

where $\mathbf{u}$ is the unique solution to the heat equation in $\Gamma$ with the same initial condition.

Also we obtain the decay of the solutions to the nonlocal problem on metric graphs, and realize that the asymptotic behaviour of those solutions is comparable to the solution of a related problem with the classical heat equation that we studied first.

Theorem 1.3. Let $1 \leq p<\infty$ and $\mathbf{u}_{0} \in L^{1}(\Gamma) \cap L^{p}(\Gamma)$. For any $1 \leq q<p$ the solution to (1.3) satisfies

$$
t^{\frac{1}{2}\left(1-\frac{1}{q}\right)}\left\|\mathbf{u}(t)-\mathbf{U}_{M}(t)\right\|_{L^{q}(\Gamma)} \rightarrow 0, \text { as } t \rightarrow \infty,
$$

where the asymptotic profile $\mathbf{U}_{M}$ is given by (1.1). 
We end this introduction with a very brief description of the methods used to prove our results. Notice that here we can not use the Fourier transform as in 14, 27] instead for the proof of the asymptotic behaviours, both for the local and the nonlocal problems, we have to rely on energy and rescaling methods, see [29, 26] and also [11. This scaling argument is delicate since it changes the graph in which the rescaled solution is defined. When we pass to the limit what remains is the unbounded part of the graph, while the finite edges collapse to a single point. This is the main reason why the asymptotic behaviour (both for the local and nonlocal cases) is given by the Gaussian profile in a half line with the total mass equally distributed according to the number of edges of infinite length. For the relaxation limit we use ideas from [1, 26] where a compactness result is proved (see the Appendix at the and of this paper).

Related works concerning shrinking of a finite part of the graph can be found in [4, 9]. Remark that in our rescaling procedure every edge of the graph is rescaled, while in the previously mentioned references only some edges are changed.

The paper is organized as follows: first, in Section 2 we introduce some notations and include the definition of the Laplcian in the graph; in Section 3 we collect more precise statements of the main results of this paper (making clear the meaning of $\sim$ and the hypothesis on the initial datum in Theorems 1.1 and 1.3). The proofs are postponed to the next two sections. Section 4 is devoted to the study of the local problem (the heat equation), and in Section [5] we tackle the nonlocal problem proving the relaxation limit and the asymptotic behaviour. Finally, Section 6 is an appendix where we include a compactness lemma that is needed when studying the relaxation limit for the nonlocal equation.

\section{Notations AND BASIC DEFINITIONS}

Before we present our results, we need to introduce some notations and basic definitions on metric graphs that we will use along the paper. Let $\Gamma=(V, E)$ be a graph where $V$ is a set of vertices and $E$ the set of edges. For each $v \in V$ we denote $E_{v}=\{e \in E: v \in e\}$. We assume that $\Gamma$ is a finite connected graph. The edges could be of finite length and then their ends are vertices of $V$ or they have infinite length and then we assume that each infinite edge is a ray with a single vertex belonging to $V$ (see e.g. [35] for more details on graphs with infinite edges). Here we assume that we do not have any terminal vertices, that is, vertices with degree one, and if we have a vertex with degree two, then we just remove it from our graph. Therefore, we will always assume that the degree of each vertex is greater or equal than three.

We fix an orientation of $\Gamma$, that is, each edge $e$ is oriented. Given an edge $e$, we denote by $I(e)$ the initial vertex and by $T(e)$ the terminal one. In the case of infinite edges we have only initial vertices. We identify every edge $e$ of $\Gamma$ with an interval $I_{e}$, where $I_{e}=\left[0, l_{e}\right]$ if the edge is finite and $I_{e}=[0, \infty)$ if the edge is infinite. This identification introduces a coordinate $x_{e}$ along the edge $e$. With this in mind we define a metric in $\Gamma, d(x, y)$ stands for the distance between two points in the graph, that is given by the length of the minimal path that joins them. In case both $x$ and $y$ belong to the same edge we have $d(x, y)=|x-y|$, when they belong to adjacent edges, $d(x, y)=|x-e|+|y-e|$ with $e$ the vertex that is 
common to the two adjacent edges, when the minimal path involve three edges we have $d(x, y)=\left|x-e_{1}\right|+\left|e_{1}-e_{2}\right|+\left|y-e_{2}\right|$, etc. In the special case when the graph is star shaped (only one vertex and $N$ edges), we have

$$
d(x, y)= \begin{cases}|x-y|, & x, y \in I_{k}, k=1, \ldots, N \\ |x|+|y|, & x \in I_{k}, y \in I_{j}, k \neq j .\end{cases}
$$

In this way $\Gamma$ is a metric space that is often named as a metric graph, see e.g. [35]. Moreover, we can write

$$
\Gamma=\Gamma_{f} \cup \Gamma_{\infty}
$$

where $\Gamma_{f}$ is made from the finite length edges of the graph $\Gamma$ whereas $\Gamma_{\infty}$ collects all the infinite edges. In the special case in which there is only one infinite edge $\Gamma_{\infty}$ is just a half line. Let $v$ be a vertex of $V$ and $e$ be an edge in $E_{v}$. We set for finite edges $e$

$$
j(v, e)=\left\{\begin{array}{lll}
0 & \text { if } & v=I(e), \\
l_{e} & \text { if } \quad v=T(e)
\end{array}\right.
$$

and

for infinite edges.

$$
j(v, e)=0, \text { if } v=I(e)
$$

We identify any function $\mathbf{u}$ on $\Gamma$ with a collection $\left\{u^{e}\right\}_{e \in E}$ of functions $u^{e}$ defined on the edges $e$ of $\Gamma$. Each $u^{e}$ can be considered as a function on the interval $I_{e}$. We will use the notation $\mathbf{u}$ to denote a function in the whole graph $\Gamma$ and $u^{e}$ to denote the function on the edge $e$. In fact, with some abuse in the notation, we will use the same notation $u^{e}$ for both the function on the edge $e$ and the function on the interval $I_{e}$ identified with $e$. For a function $\mathbf{u}: \Gamma \rightarrow \mathbb{R}, \mathbf{u}=\left\{u^{e}\right\}_{e \in E}$, and $f: \mathbb{R} \mapsto \mathbb{R}$ we denote by $f(\mathbf{u}): \Gamma \rightarrow \mathbb{R}$ the family $\left\{f\left(u^{e}\right)\right\}_{e \in E}$, where $f\left(u^{e}\right): e \rightarrow \mathbb{R}$ is given by $f\left(u^{e}\right)\left(x_{e}\right)=f\left(u^{e}\left(x_{e}\right)\right)$.

We introduce $C(\Gamma)$ the space of continuous functions on $\Gamma$. A function $\mathbf{u}=\left\{u^{e}\right\}_{e \in E}$ is continuous on $\Gamma$ if and only if $u^{e}$ is continuous on $I_{e}$ for every $e \in E$, and we have continuity at the vertices, that is,

$$
u^{e}(j(v, e))=u^{e^{\prime}}\left(j\left(v, e^{\prime}\right)\right), \quad \forall e, e^{\prime} \in E_{v} .
$$

The space $L^{p}(\Gamma), 1 \leq p<\infty$ consists of all functions $\mathbf{u}=\left\{u_{e}\right\}_{e \in E}$ on $\Gamma$ that belong to $L^{p}\left(I_{e}\right)$ for each edge $e \in E$ and we endow it with the norm

$$
\|\mathbf{u}\|_{L^{p}(\Gamma)}^{p}=\sum_{e \in E}\left\|u^{e}\right\|_{L^{p}\left(I_{e}\right)}^{p} .
$$

Similarly, the space $L^{\infty}(\Gamma)$ consists of all functions that belong to $L^{\infty}\left(I_{e}\right)$ for each edge $e \in E$ and

$$
\|\mathbf{u}\|_{L^{\infty}(\Gamma)}=\max _{e \in E}\left\|u^{e}\right\|_{L^{\infty}\left(I_{e}\right)}
$$

The Sobolev space $H^{1}(\Gamma)$, consists in all functions on $\Gamma$ that belong to $H^{1}\left(I_{e}\right)$ for each $e \in E$ and

$$
\|\mathbf{u}\|_{H^{1}(\Gamma)}^{2}=\sum_{e \in E}\left\|u^{e}\right\|_{H^{1}(e)}^{2}
$$


Sobolev spaces with higher differentiability $H^{m}(\Gamma), m \geq 2$, can be defined in an analogous way. The spaces $L^{2}(\Gamma)$ and $H^{1}(\Gamma)$ are Hilbert spaces with the inner products

$$
(\mathbf{u}, \mathbf{v})_{L^{2}(\Gamma)}=\sum_{e \in E}\left(u^{e}, v^{e}\right)_{L^{2}\left(I_{e}\right)}=\sum_{e \in E} \int_{I_{e}} u^{e}\left(x_{e}\right) v^{e}\left(x_{e}\right) d x_{e}
$$

and

$$
(\mathbf{u}, \mathbf{v})_{H^{1}(\Gamma)}=\sum_{e \in E}\left(u^{e}, v^{e}\right)_{H^{1}\left(I_{e}\right)}=\sum_{e \in E} \int_{I_{e}} \frac{d u^{e}}{d x}\left(x_{e}\right) \frac{d v^{e}}{d x}\left(x_{e}\right) d x_{e} .
$$

Notice that this defines an inner product in $H^{1}(\Gamma)$.

We now define the exterior normal derivative of a function $\mathbf{u}=\left\{u^{e}\right\}_{e \in E}$ at the endpoints of the edges. For each $e \in E$ and $v$ an endpoint of $e$ we consider the normal derivative of the restriction of $\mathbf{u}$ to the edge $e$ of $E_{v}$ evaluated at $j(v, e)$ to be defined by:

$$
\frac{\partial u^{e}}{\partial n_{e}}(j(v, e))= \begin{cases}-u_{x}^{e}(0+) & \text { if } j(v, e)=0 \\ u_{x}^{e}\left(l_{e}-\right) & \text { if } j(v, e)=l_{e}\end{cases}
$$

We now introduce the Laplace operator $\Delta_{\Gamma}$ on the graph $\Gamma$. This is a standard procedure and we refer the interested reader to [15]. The operator $\Delta_{\Gamma}$ has domain

$$
D\left(\Delta_{\Gamma}\right)=\left\{\mathbf{u}=\left\{u^{e}\right\}_{e \in E} \in H^{2}(\Gamma): \mathbf{u} \text { continuous and } \sum_{e \in E_{v}} \frac{\partial u^{e}}{\partial n_{e}}(j(v, e))=0 \quad \text { for all } v \in V\right\}
$$

and it applies to any function $\mathbf{u} \in D\left(\Delta_{\Gamma}\right)$ as follows

$$
\left(\Delta_{\Gamma} \mathbf{u}\right)^{e}=\left(u^{e}\right)_{x x} \quad \text { for all } e \in E .
$$

In other words, $D\left(\Delta_{\Gamma}\right)$ is the space of all continuous functions on $\Gamma, \mathbf{u}=\left\{u^{e}\right\}_{e \in E}$, such that for every edge $e \in E, u^{e} \in H^{2}\left(I_{e}\right)$, and the following Kirchhoff-type condition is satisfied,

$$
\sum_{e \in E: T(e)=v} u_{x}^{e}\left(l_{e}-\right)-\sum_{e \in E: I(e)=v} u_{x}^{e}(0+)=0 \quad \text { for all } v \in V .
$$

In the particular case when $\Gamma$ consists on only one edge these conditions reduce to the classical Laplacian with Neumann boundary conditions.

The quadratic form associated to $\Delta_{\Gamma}$ is given by

$$
\mathcal{Q}_{\Gamma}(\mathbf{u}, \mathbf{u})=\left(\mathbf{u}_{x}, \mathbf{u}_{x}\right)_{L^{2}(\Gamma)}
$$

for all $\mathbf{u} \in D\left(\mathcal{Q}_{\Gamma}\right)$ where

$$
D\left(\mathcal{Q}_{\Gamma}\right)=D\left(\left(-\Delta_{\Gamma}\right)^{1 / 2}\right)=\left\{\mathbf{u}=\left\{u^{e}\right\}_{e \in E} \in H^{1}(\Gamma): \mathbf{u} \text { continuous }\right\} .
$$

In particular $D\left(\mathcal{Q}_{\Gamma}\right)$ with the $H^{1}(\Gamma)$-norm is a Banach space and $\mathcal{Q}_{\Gamma}(\mathbf{u}, \mathbf{u})=-\left(\mathbf{u}, \Delta_{\Gamma} \mathbf{u}\right)$ for all $\mathbf{u} \in D\left(\Delta_{\Gamma}\right)$. For other kinds of Laplace operators on metric graphs, we refer to [3, 33].

It is easy to verify that $\left(\Delta_{\Gamma}, D\left(\Delta_{\Gamma}\right)\right)$ is a linear, unbounded, self-adjoint, dissipative operator on $L^{2}(\Gamma)$, i.e. $\left(\Delta_{\Gamma} \mathbf{u}, \mathbf{u}\right)_{L^{2}(\Gamma)} \leq 0$ for all $\mathbf{u} \in D\left(\Delta_{\Gamma}\right)$. Since $\bigoplus_{e \in \Gamma} C_{c}^{\infty}\left(I_{e}\right) \subset D\left(\Delta_{\Gamma}\right)$ we obtain that $D\left(\Delta_{\Gamma}\right)$ is dense in any $L^{p}(\Gamma), 1 \leq p<\infty$. The analysis of the operator $\Delta_{\Gamma}$ on $L^{p}(\Gamma)$-spaces will be discussed in Section 4 . 
The main results of this paper are proved by rescaling the solutions with a parameter that we will call $\lambda$, considering $\mathbf{u}_{\lambda}(x)=\lambda \mathbf{u}(\lambda x)$. Since the equation is defined on edges that are either finite or infinite we have to rescale properly not only the solutions of our equations but also their domain of definition $\Gamma^{\lambda}=\left(V, E^{\lambda}\right)$. The graph $\Gamma^{\lambda}$ is obtained from $\Gamma=(V, E)$ by rescaling properly the intervals $I_{e}^{\lambda}$ that parametrize the edges $E^{\lambda}$ of the graph $\Gamma^{\lambda}$. We set

$$
I_{e}^{\lambda}= \begin{cases}{\left[0, l_{e} / \lambda\right],} & \text { if } l_{e}<\infty, \\ {[0, \infty),} & \text { if } l_{e}=\infty .\end{cases}
$$

In this way we have $\Gamma^{\lambda}=\Gamma_{f}^{\lambda} \cup \Gamma_{\infty}$ since $\Gamma_{\infty}$ remains invariant under the above transformation.

\section{Main Results}

In this section we collect the statements of the main results of this manuscript. We postpone their proofs to the next sections. We also write related literature and motivation to research in local and nonlocal evolution problems on metric graphs.

3.1. The local evolution problem. Associated with the Laplacian we consider the heat equation on $\Gamma$ :

$$
\begin{cases}\mathbf{u}_{t}(t, x)-\Delta_{\Gamma} \mathbf{u}(t, x)=0, & x \in \Gamma, t>0, \\ \mathbf{u}(0, x)=\mathbf{u}_{0}(x), & x \in \Gamma .\end{cases}
$$

We point out that the well-posedness for this problem is a direct consequence of Hille-YosidaPhillips theorem. For example if the initial data $\mathbf{u}_{0}$ belongs to $L^{1}(\Gamma)$ then there exists a unique solution $\mathbf{u} \in C\left([0, \infty), L^{1}(\Gamma)\right)$ and it satisfies

$$
\int_{\Gamma} \mathbf{u}(t, x) d x=\int_{\Gamma} \mathbf{u}_{0}(x) d x, \quad\|\mathbf{u}(t)\|_{L^{1}(\Gamma)} \leq\left\|\mathbf{u}_{0}\right\|_{L^{1}(\Gamma)} .
$$

Moreover, the ultracontractivity of the semigroup generated by the operator $\Delta_{\Gamma}$ gives us that the solution $\mathbf{u}$ also decays when the time increases

$$
\|\mathbf{u}(t)\|_{L^{p}(\Gamma)} \leq C(p, \Gamma) t^{-\frac{1}{2}\left(1-\frac{1}{p}\right)}\left\|\mathbf{u}_{0}\right\|_{L^{1}(\Gamma)}, \quad 1 \leq p \leq \infty .
$$

More details about the well-poesedness of problem (3.4) will be given in Section 4 .

Starting from these estimates and using a rescaling procedure we can prove that for a connected graph with a finite number of edges the long time behaviour of the solution is given by the same problem in the star-shaped tree that is obtained when the bounded segments, the compact core of the graph, collapse to a single point. In this case the asymptotic profile is given by the solution to the classical heat equation in the half line with Neumann boundary condition at $x=0$ and as initial datum at $t=0$ a Dirac mass at $x=0$. This last solution is self similar with a well known Gaussian profile. To obtain the limit profile for our evolution problem, we just repeat this Gaussian profile in any infinite line and multiply by $2 M / N$, being $M$ the total mass, and $N$ the number of infinity length segments.

Theorem 3.1. Let $\mathbf{u}_{0} \in L^{1}(\Gamma)$ and $\mathbf{u}$ the solution of the problem (3.4). Then for any $1 \leq p \leq \infty$

$$
t^{\frac{1}{2}\left(1-\frac{1}{p}\right)}\left\|\mathbf{u}(t)-\mathbf{U}_{M}(t)\right\|_{L^{p}\left(\Gamma_{\infty}\right)} \rightarrow 0, \quad \text { as } t \rightarrow \infty
$$


and

$$
t^{\frac{1}{2}}\left\|\mathbf{u}(t)-\mathbf{U}_{M}(t)\right\|_{L^{p}\left(\Gamma_{f}\right)} \rightarrow 0, \quad \text { as } t \rightarrow \infty
$$

where $M$ is the total mass of the initial datum $\mathbf{u}_{0}$ and

$$
\mathbf{U}_{M}(x, t)=\frac{2 M}{N} t^{-\frac{1}{2}} \begin{cases}G(x / \sqrt{t}), & x \in \Gamma_{\infty}, \\ G(0), & x \in \Gamma_{f} .\end{cases}
$$

3.2. The nonlocal evolution problem. Now, let us look at nonlocal equations on a metric graph $\Gamma$. We consider the evolution problem,

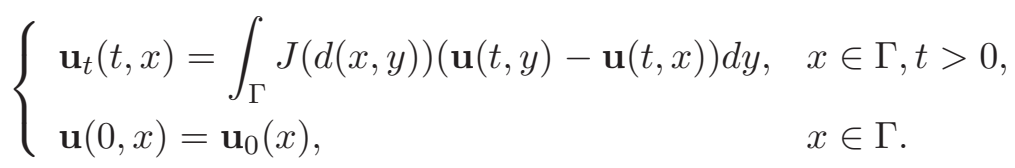

The kernel $J \in L^{1}(\mathbb{R}) \cap L^{1}\left(\mathbb{R},|x|^{2}\right)$ is assumed to be symmetric, $J(z)$ is non-increasing for $z>0, J$ is nonnegative in $\mathbb{R}$ and positive in neighborhood of the origin. Notice that we are not assuming the solution $\mathbf{u}$ to the nonlocal evolution problem (3.9) to be continuous, and hence, we do not impose any condition on the vertices.

The assumption that $J \in L^{1}(\mathbb{R})$ guarantees that operator

$$
\mathbf{u} \mapsto L(\mathbf{u}):=\int_{\Gamma} J(d(x, y))(\mathbf{u}(y)-\mathbf{u}(x)) d y
$$

is bounded between any $L^{p}(\Gamma)$ spaces, $1 \leq p \leq \infty$. It generates a contraction semigroup in these spaces which is positive preserving and Markovian. Results about the well posedness are given in Theorem 5.1 .

Both local and nonlocal models are similar in the sense that they share some properties such as existence and uniqueness of solutions, conservation of the total mass of the initial datum and the validity of the strong maximum principle. However, solutions to the nonlocal problem do not have a regularizing effect in time. Solutions are as smooth in space for $t>0$ as the initial data are (this fact is due to the integrability of the kernel $J$ ). If $\mathbf{u}_{0}$ belongs to a suitable space $X$, then $\mathbf{u}(t) \in X$ for all times and it is no better (there is no regularizing effect in strong contrast with the local heat equation). This lack of smoothing is a major difficulty for the analysis of the asymptotic behaviour of solutions since it implies some lack of compactness of the trajectories, $\{\mathbf{u}(t)\}_{t>0}$. Since there is a lack of regularizing effect we cannot analyze the ultracontractivity property in the classical sense ([19]): for initial data in $L^{1}(\Gamma) \backslash L^{\infty}(\Gamma)$ the semigroup is not in $L^{\infty}(\Gamma)$ at any positive time. However, we can prove that for initial data in $L^{1}(\Gamma) \cap L^{p}(\Gamma)$ the solution has a certain decay in the $L^{p}(\Gamma)$ norm, see Theorem 5.2.

3.2.1. A relaxation limit. First, we establish one more evidence to support that the nonlocal problem (3.9) and the local heat equation (3.4) are closely related. To this end let us consider the following relaxation problem: for each $\varepsilon>0$ consider the problem

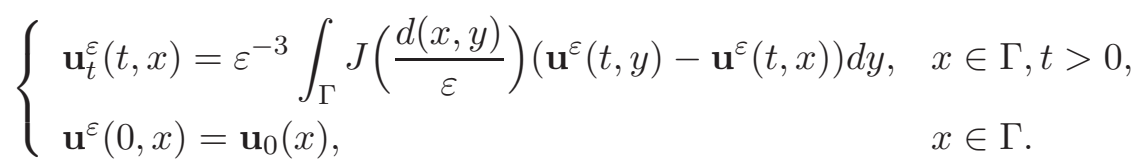


and analyze the limit problem when $\varepsilon \rightarrow 0$. We recall that to simplify the presentation we assume that

$$
\frac{1}{2} \int_{\mathbb{R}} z^{2} J(z) d z=1
$$

Theorem 3.2. For any $\mathbf{u}_{0} \in L^{2}(\Gamma)$ it holds that

$$
\mathbf{u}^{\varepsilon} \rightarrow \mathbf{u} \text { weakly in } L^{2}\left([0, \infty), L^{2}(\Gamma)\right),
$$

where $\mathbf{u} \in C\left([0, \infty), L^{2}(\Gamma)\right)$ is the unique solution of the heat equation (3.4) with the same initial condition.

Notice that, when $u$ is a smooth function, a simple Taylor expansion gives

$$
\varepsilon^{-3} \int_{I} J\left(\frac{|x-y|}{\varepsilon}\right)(u(y)-u(x)) d y \approx\left(\frac{1}{2} \int_{\mathbb{R}} J(z) z^{2} d x\right) u_{x x}(x)
$$

for $x$ an interior point of an interval $I$. What is remarkable here is that in the limit as $\varepsilon \rightarrow 0$ we recover the Kirchoff conditions on the nodes without assuming any condition on $\mathbf{u}^{\varepsilon}$ (not even continuity). For other relaxation limits of this kind we refer to [18, 37] and the book [1] and references therein.

It will be interesting to analyze under which conditions on function $J$ we can recover in the relaxation limit more general operators $\Delta_{A, B}$ like the ones described in [33, [3]. These acts exactly as the laplacian on the edges but there are different coupling conditions at the vertices of the type $A \mathbf{u}^{\prime}(v)+B \mathbf{u}(v)=0$ with suitable conditions under the matrices $A$ and $B$. This will be investigated in a future work.

3.2.2. Asymptotic behaviour for the nonlocal problem. In spite of this lack of regularizing effect, in the one dimensional case, when the problem is posed in the whole $\mathbb{R}$, is it shown in [14 that solutions to the equation

$$
u_{t}(t, x)=\int_{\mathbb{R}} J(x-y)(u(t, y)-u(t, x)) d y
$$

have the same asymptotic behaviour as solutions to the classical heat equation $u_{t}=u_{x x}$. Therefore, we expect that solutions to (3.9) in the graph also have the same asymptotic behaviour (the same decay bounds (3.5) and asymptotic profile) as solutions to the heat equation, (3.4), that we analyzed first in Theorem 3.1.

Theorem 3.3. Let $1 \leq p<\infty$. For any $\mathbf{u}_{0} \in L^{1}(\Gamma) \cap L^{p}(\Gamma)$ the solution to (3.9) satisfies

$$
\|\mathbf{u}(t)\|_{L^{p}(\Gamma)} \leq \frac{C\left(\left\|\mathbf{u}_{0}\right\|_{L^{1}(\Gamma)},\left\|\mathbf{u}_{0}\right\|_{L^{p}(\Gamma)}\right)}{(1+t)^{\frac{1}{2}\left(1-\frac{1}{p}\right)}}, \quad \forall t>0 .
$$

For any $1 \leq q<p$ the asymptotic profile $\mathbf{U}_{M}$ is given by (3.8):

$$
t^{\frac{1}{2}\left(1-\frac{1}{q}\right)}\left\|\mathbf{u}(t)-\mathbf{U}_{M}(t)\right\|_{L^{q}(\Gamma)} \rightarrow 0, \text { as } t \rightarrow \infty .
$$

Remark 3.1. The asymptotic profile is the same as the one that we obtained for the local problem. If we don't assume $\frac{1}{2} \int_{\mathbb{R}} z^{2} J(z) d z=1$, we just let $0<A=\frac{1}{2} \int_{\mathbb{R}} z^{2} J(z) d z<+\infty$ and we obtain that the asymptotic profile $\mathbf{U}_{A, M}$ verifies $\mathbf{U}_{t}=A \Delta_{\Gamma} \mathbf{U}$ and $\mathbf{U}(0)=M \delta_{0}$ so 
$\mathbf{U}_{A, M}(t, x)=\mathbf{U}_{M}(A t, x)$. The convergence of the compact part of the graph can be slightly improved in the following sense: for a fixed $1<p^{*}<p$ the following holds for any $1 \leq q \leq p^{*}$ :

$$
t^{\frac{1}{2}\left(1-\frac{1}{p^{*}}\right)}\left\|\mathbf{u}(t)-\mathbf{U}_{M}(t)\right\|_{L^{q}\left(\Gamma_{f}\right)} \rightarrow 0, \text { as } t \rightarrow \infty .
$$

\section{Asymptotic Behaviour for the heat equation}

Let us consider the heat equation on $\Gamma$ :

$$
\begin{cases}\mathbf{u}_{t}(t, x)-\Delta_{\Gamma} \mathbf{u}(t, x)=0, & x \in \Gamma, t>0, \\ \mathbf{u}(0, x)=\mathbf{u}_{0}(x), & x \in \Gamma .\end{cases}
$$

In this section we show that for a general graph the long time behaviour of the solution is given by the problem in a star-shaped tree that is obtained from $\Gamma$ when the bounded segments, the compact core of the graph, collapse to a single point. In this case the asymptotic profile is given by the solution in the half line with Neumann boundary condition at $x=0$ and initial data a Dirac mass at $x=0$ multiplied by an adequate constant that takes into account the number of infinite edges and the mass of the initial condition (we multiply by $2 M / N$, being $M$ the total mass, and $N$ the number of infinit length segments).

We now present few well know facts about the semigroup generated by the Laplacian on metric graphs. For the sake of completeness we prefer to include here some details for the interested reader. In the $L^{2}$-setting, using the fact that $\left(\Delta_{\Gamma}, D\left(\Delta_{\Gamma}\right)\right)$ is maximal dissipative we easily obtain that it generates a strongly continuous semigroup of contractions in $L^{2}(\Gamma)$ that we denote by $S(t)$. This means that for any $\mathbf{u}_{0} \in D\left(\Delta_{\Gamma}\right)$ there exists a unique solution $\mathbf{u}(t)=$ $S(t) \mathbf{u}_{0}$ of system (4.1) that satisfies $\mathbf{u} \in C\left([0, \infty), D\left(\Delta_{\Gamma}\right)\right) \cap C^{1}\left([0, \infty), L^{2}(\Gamma)\right)$. Moreover since $\Delta_{\Gamma}$ is self adjoint we also have more regularity on the solution, in particular $\mathbf{u} \in$ $C^{\infty}\left((0, \infty), D\left(\Delta_{\Gamma}\right)\right)$. Thus, for any $\mathbf{u}_{0} \in D\left(\Delta_{\Gamma}\right)$ system (4.1) can be written in an explicit way as follows:

$$
\begin{cases}u^{e} \in C\left([0, \infty), H^{2}\left(I_{e}\right)\right) \cap C^{1}\left([0, \infty), L^{2}\left(I_{e}\right)\right), & e \in E, \\ u_{t}^{e}(t, x)-u_{x x}^{e}(t, x)=0, & x \in I_{e}, t>0, \\ \text { for all } v \in V, u^{e}(t, j(v, e))=u^{e^{\prime}}\left(t, j\left(v, e^{\prime}\right)\right), & \forall e, e^{\prime} \in E_{v}, t>0, \\ \sum_{e \in E: T(e)=v} u_{x}^{e}\left(t, l_{e}-\right)-\sum_{e \in E: I(e)=v} u_{x}^{e}(t, 0+)=0, & \text { for all } v \in V .\end{cases}
$$

In the context of the $L^{p}$-spaces, $1 \leq p<\infty$ there are several papers on the subject. We refer to recent paper [20] where using a direct method it is proved that more general second order operators under more general conditions generate a strongly continuous semigroup of contractions in $L^{p}(\Gamma), 1 \leq p<\infty$. In particular, $\Delta_{\Gamma}$ with Kirchhoff conditions fulfill the hypotheses in the previously mentioned reference [20]. Other approaches are possible. For example, if one starts from the $L^{2}$-theory and then use interpolation theory one can obtain similar results. We cite here [38] and [34] where the authors treat graphs with finitely many edges of finite length but general couplings. Their method allow to first prove that the $L^{2}-$ semigroup is positive and contractive in $L^{\infty}(\Gamma)$. The proof relays on the characterization in 
[39] based on Kato-type inequalities, i.e.

$$
\left(\Delta_{\Gamma} \mathbf{u},(|\mathbf{u}|-1)^{+} \operatorname{sgn}(\mathbf{u})\right) \leq 0, \quad \forall \mathbf{u} \in D\left(\Delta_{\Gamma}\right) .
$$

Observe that when $\left\|\mathbf{u}_{0}\right\|_{L^{\infty}(\Gamma)} \leq 1$ and we try to show that the solution satisfies $\|\mathbf{u}(t)\|_{L^{\infty}(\Gamma)} \leq$ 1 for all positive time, one derivative in time of $\left\|(\mathbf{u}(t)-1)^{+}\right\|_{L^{2}(\Gamma)}^{2}$ leads to

$\frac{1}{2} \int_{\Gamma}\left[(\mathbf{u}(t, x)-1)^{+}\right]^{2} d x=\int_{\Gamma} \mathbf{u}_{t}(t, x)(\mathbf{u}(t, x)-1)^{+} \operatorname{sgn}(\mathbf{u}(t, x)) d x=\left(\Delta_{\Gamma} \mathbf{u},(|\mathbf{u}|-1)^{+} \operatorname{sgn}(\mathbf{u})\right)$ and inequality (4.3) gives the desired result.

A classical argument (see [39, p. 56]) involving Riesz-Thorin interpolation theory shows that $S(t)=e^{t \Delta_{\Gamma}}$ can be extended to a strongly continuous semigroup of contractions in $L^{p}(\Gamma)$ for each $2 \leq p<\infty$. By duality $e^{t \Delta_{\Gamma}^{*}}$ is strongly continuous and contractive for $1<p \leq 2$. An approximation on compact sets shows that it is also in $L^{1}(\Gamma)$. This also follow arguing as in [2, Section 7.2.1].

A special attention has been paid to the $L^{1}-L^{\infty}$ estimates of the semigroup. In [25], see also [38. for compact graphs, it has been proved that

$$
\|\mathbf{u}\|_{L^{2}(\Gamma)} \leq C\left\|\mathbf{u}_{x}\right\|_{L^{2}(\Gamma)}^{1 / 3}\|\mathbf{u}\|_{L^{1}(\Gamma)}^{2 / 3}
$$

holds for a graph having some infinite edges attached to the compact part of the graph. In view of [19] these estimates allow to prove the ultracontractivity of the semigroup, i.e.

$$
\left\|e^{t \Delta_{\Gamma}} \varphi\right\|_{L^{\infty}(\Gamma)} \leq C t^{-1 / 4}\|\varphi\|_{L^{2}(\Gamma)}, \quad \forall \varphi \in L^{2}(\Gamma) .
$$

Duality arguments give us a similar $L^{1}-L^{2}$ estimates, which together with the previous one show an $L^{1}-L^{\infty}$ estimate on the semigroup. This shows that for any $\varphi \in L^{1}(\Gamma)$ the solution u belongs to $C\left((0, \infty), L^{p}(\Gamma)\right)$ for any $1 \leq p<\infty$. In particular we can use the $L^{2}$-theory for $t>t_{0}$ with $t_{0}>0$ arbitrary to obtain regularity properties for the solution and justify all the formal computations done in the rest of the paper.

Therefore, the following existence and uniqueness result holds.

Theorem 4.1. For any $\mathbf{u}_{0} \in D\left(\Delta_{\Gamma}\right)$ there exists a unique solution $\mathbf{u}(t)$ of system (4.1) that satisfies $\mathbf{u} \in C\left([0, \infty), D\left(\Delta_{\Gamma}\right)\right) \cap C^{1}\left([0, \infty), L^{2}(\Gamma)\right)$. Moreover, for any $\mathbf{u}_{0} \in L^{p}(\Gamma), 1 \leq p<\infty$, there exists a unique solution $\mathbf{u} \in C\left([0, \infty), L^{p}(\Gamma)\right)$ that satisfies

$$
\|\mathbf{u}(t)\|_{L^{p}(\Gamma)} \leq\left\|\mathbf{u}_{0}\right\|_{L^{p}(\Gamma)} \quad \text { for all } t \geq 0 .
$$

Remark 4.1. For any $\mathbf{u}_{0} \in D\left(\Delta_{\Gamma}\right) \cap L^{1}(\Gamma)$, there exists a unique solution $\mathbf{u} \in C\left([0, \infty), D\left(\Delta_{\Gamma}\right) \cap\right.$ $\left.L^{1}(\Gamma)\right)$.

In the next result we obtain some decay bounds for the solutions.

Theorem 4.2. For any $\mathbf{u}_{0} \in L^{1}(\Gamma)$ the solution of system (4.1) satisfies

$$
\begin{gathered}
\|\mathbf{u}(t)\|_{L^{1}(\Gamma)} \leq\left\|\mathbf{u}_{0}\right\|_{L^{1}(\Gamma)}, \\
\int_{\Gamma} \mathbf{u}(t, x) d x=\int_{\Gamma} \mathbf{u}_{0}(x) d x, \\
\|\mathbf{u}(t)\|_{L^{p}(\Gamma)} \leq C(p, \Gamma) t^{-\frac{1}{2}\left(1-\frac{1}{p}\right)}\left\|\mathbf{u}_{0}\right\|_{L^{1}(\Gamma)}, 1 \leq p \leq \infty,
\end{gathered}
$$




$$
\left\|\mathbf{u}_{x}(t)\right\|_{L^{2}(\Gamma)} \leq C(\Gamma) t^{-\frac{3}{2}}\left\|\mathbf{u}_{0}\right\|_{L^{1}(\Gamma)}
$$

and an energy estimate

$$
\int_{0}^{T} \int_{\Gamma} \mathbf{u}_{x}^{2}(t, x) d x d t=\|\mathbf{u}(0)\|_{L^{2}(\Gamma)}^{2}-\|\mathbf{u}(t)\|_{L^{2}(\Gamma)}^{2} .
$$

Proof. The first property is the contractivity in $L^{1}(\Gamma)$ of the semigroup discussed above. The mass conservation follows by considering initial data $\mathbf{u}_{0} \in D\left(\Delta_{\Gamma}\right) \cap L^{1}(\Gamma)$, proving the property for these solutions and finally using an approximation argument. Indeed when the solution is regular we integrate in space variable equation (4.2) to obtain that the mass is constant in time. Indeed, since $\mathbf{u}(t) \in D\left(\Delta_{\Gamma}\right)$ and $\mathbf{1} \in D\left(\mathcal{Q}_{\Gamma}\right)$

$$
\frac{d}{d t} \int_{\Gamma} \mathbf{u}(t, x) d x=\left(\Delta_{\Gamma} \mathbf{u}, 1\right)=-\mathcal{Q}\left(\mathbf{u}_{x}, \mathbf{1}_{x}\right)=0
$$

The third property follows from [25, Th. 2.].

The estimate on the derivative of $\mathbf{u}$ follows since $\Delta_{\Gamma}$ is self-adjoint (see [13, Section 3.2, p.35]). Indeed, for any $\mathbf{v}_{0} \in L^{2}(\Gamma)$ we have that $S(t)$, the semigroup generated by $\Delta_{\Gamma}$, satisfies $S(t) \mathbf{v}_{0} \in D\left(\Delta_{\Gamma}\right)$ for any $t>0$ and

$$
\left\|\Delta_{\Gamma} S(t) \mathbf{v}_{0}\right\|_{L^{2}(\Gamma)} \leq t^{-1}\left\|\mathbf{v}_{0}\right\|_{L^{2}(\Gamma)} .
$$

Using this property with $\mathbf{v}_{0}=S(t) \mathbf{u}_{0}$ and estimate (4.4) we obtain

$$
\left\|\Delta_{\Gamma} S(2 t) \mathbf{u}_{0}\right\|_{L^{2}(\Gamma)} \leq t^{-1}\left\|S(t) \mathbf{u}_{0}\right\|_{L^{2}(\Gamma)} \leq C(\Gamma) t^{-1} t^{-\frac{1}{4}}\left\|\mathbf{u}_{0}\right\|_{L^{1}(\Gamma)}=C(\Gamma) t^{-\frac{5}{4}}\left\|\mathbf{u}_{0}\right\|_{L^{1}(\Gamma)} .
$$

Now, since $S(t) \mathbf{u}_{0} \in D\left(\Delta_{\Gamma}\right)$ for any $t>0$ we get

$$
\left\langle\partial_{x}\left(S(t) \mathbf{u}_{0}\right), \partial_{x}\left(S(t) \mathbf{u}_{0}\right)\right\rangle=\mathcal{Q}_{\Gamma}\left(S(t) \mathbf{u}_{0}, S(t) \mathbf{u}_{0}\right)=\left\langle S(t) \mathbf{u}_{0}, \Delta_{\Gamma}\left(S(t) \mathbf{u}_{0}\right)\right\rangle .
$$

Using the above inequalities on the semigroup we obtain

$$
\left\|\partial_{x}\left(S(t) \mathbf{u}_{0}\right)\right\|_{L^{2}(\Gamma)}^{2} \leq\left\|S(t) \mathbf{u}_{0}\right\|_{L^{2}(\Gamma)} \| \Delta_{\Gamma}\left(S(t) \mathbf{u}_{0}\left\|_{L^{2}(\Gamma)} \leq t^{-\frac{1}{4}}\right\| \mathbf{u}_{0}\left\|_{L^{1}(\Gamma)} t^{-\frac{5}{4}}\right\| \mathbf{u}_{0} \|_{L^{1}(\Gamma)},\right.
$$

which gives us the desired estimate.

Now we are ready to proceed with the proof of our main result in this section, namely Theorem 3.1 .

In order to prove this result we use the method of rescaling the solutions. For any solution $\mathbf{u}$ of system (4.1) we introduce the family $\mathbf{u}_{\lambda}: \Gamma^{\lambda} \rightarrow \mathbb{R}, \lambda>0$ :

$$
\mathbf{u}_{\lambda}(t, x)=\lambda \mathbf{u}\left(\lambda^{2} t, \lambda x\right), \quad x \in \Gamma^{\lambda}, t>0 .
$$

It is immediate to see that $\mathbf{u}_{\lambda}$ satisfies the system

$$
\begin{cases}\mathbf{u}_{\lambda, t}(t, x)-\Delta_{\Gamma^{\lambda}} \mathbf{u}_{\lambda}(t, x)=0, & x \in \Gamma^{\lambda}, t \neq 0, \\ \mathbf{u}_{\lambda}(0, x)=\mathbf{u}_{0 \lambda}(x), & x \in \Gamma^{\lambda}\end{cases}
$$

If $\mathbf{u}_{0} \in L^{1}(\Gamma) \cap D\left(\Delta_{\Gamma}\right)$ the rescaled solution $\mathbf{u}_{\lambda}$ satisfies a similar equation as (4.2) but on the rescaled intervals $I_{e}^{\lambda}$ instead of $I_{e}$. 
First, we need to obtain some bounds. From now on we will look for constants that are independent of $\lambda$. We will use the standard notation $\lesssim$ to denote a less or equal bound in which the involved constant does not depend on the relevant quantities.

Lemma 4.1. For any $\mathbf{u}_{0} \in L^{1}(\Gamma)$ the family $\left\{\mathbf{u}_{\lambda}\right\}_{\lambda>0}$ satisfies the following uniform estimates

$$
\begin{gathered}
\int_{\Gamma} \mathbf{u}_{\lambda}(t, x) d x=\int_{\Gamma} \mathbf{u}_{0}(x) d x \\
\left\|\mathbf{u}_{\lambda}(t)\right\|_{L^{p}(\Gamma)} \leq C(p, \Gamma) t^{-\frac{1}{2}\left(1-\frac{1}{p}\right)}\left\|\mathbf{u}_{0}\right\|_{L^{1}(\Gamma)},
\end{gathered}
$$

and

$$
\left\|\mathbf{u}_{\lambda, x}(t)\right\|_{L^{2}(\Gamma)} \leq C(p, \Gamma) t^{-\frac{3}{4}}\left\|\mathbf{u}_{0}\right\|_{L^{1}(\Gamma)} .
$$

Moreover, for any $\tau>0$,

$$
\int_{\tau}^{T} \int_{\Gamma} \mathbf{u}_{\lambda, x}^{2}(t, x) d x d t \leq C(\Gamma) \tau^{-\frac{1}{2}}\left\|\mathbf{u}_{0}\right\|_{L^{1}(\Gamma)}^{2} .
$$

Finally, there exists a positive constant $C$ such that for any $\lambda>1$,

$$
\int_{\Gamma_{\infty},|x|>2 R}\left|\mathbf{u}_{\lambda}(t, x)\right| d x \leq \int_{\Gamma_{\infty},|x|>R}\left|\mathbf{u}_{0}(x)\right| d x+\frac{C M t}{R^{2}} .
$$

Proof. The first three estimates follow directly from Theorem 4.2. The forth bound follows by using the energy identity (4.6) with initial data at time $\tau$ and the uniform decay of the $L^{2}$-norm of $\mathbf{u}_{\lambda}$.

We prove estimate (4.11) for nonnegative solutions since the general case follows immediately. Let us consider $u_{\lambda}^{1}, \ldots, u_{\lambda}^{N}$ the restriction of $\mathbf{u}_{\lambda}$ to $\Gamma_{\infty}$. We will prove the desired estimate only for $u_{\lambda}^{1}$ since the others cases are similar. Summing the estimates for each $u_{\lambda}^{k}$ gives us the desired estimate.

Let us consider a smooth function $\psi:(0, \infty) \rightarrow \mathbb{R}$ such that $0 \leq \psi \leq 1$ and $\psi \equiv 0$ in $(0,1)$ and $\psi \equiv 1$ in $x \in(2, \infty)$. We define $\psi_{R}(x)=\psi(x / R)$. Multiplying the equation satisfied by $u_{\lambda}^{1}$ with $\psi_{R}$ and integrating in space and time we obtain:

$$
\begin{aligned}
\int_{0}^{\infty} u_{\lambda}^{1}(t, x) \psi_{R}(x) d x & =\int_{0}^{\infty} u_{\lambda}^{1}(0, x) \psi_{R}(x) d x+\int_{0}^{t} \int_{0}^{\infty} u_{\lambda}^{1}(s, x)\left(\psi_{R}\right)_{x x}(x) d x d s \\
& \leq \int_{0}^{\infty} u_{\lambda}^{1}(0, x) \psi_{R}(x) d x+\frac{M t}{R^{2}}\left\|\psi^{\prime \prime}\right\|_{L^{\infty}((0, \infty))} \\
& \leq \int_{\lambda R}^{\infty} u^{1}(0, x) \psi_{R}(x / \lambda) d x+\frac{M t}{R^{2}}\left\|\psi^{\prime \prime}\right\|_{L^{\infty}((0, \infty))} .
\end{aligned}
$$

Since $\psi_{R} \equiv 1$ on $(2 R, \infty)$ and $\lambda>1$ we obtain

$$
\int_{2 R}^{\infty} u_{\lambda}^{1}(t, x) d x \leq \int_{R}^{\infty} u_{0}^{1}(x)+\frac{C M t}{R^{2}}
$$

which finishes the proof. 
Proof of Theorem 3.1. We divide the proof in several steps. We will prove the results for $\mathbf{u}_{0} \in L^{1}(\Gamma) \cap D\left(\Delta_{\Gamma}\right)$ and then by an approximation argument we will obtain the result for $L^{1}$-initial data.

Step I. Compactness of the family $\mathbf{u}_{\lambda}$. We prove that up to a subsequence $\lambda_{j} \rightarrow \infty$ the family $\left\{\mathbf{u}_{\lambda}\right\}_{\lambda>0}$ converges to some function $\mathbf{U}$.

Let us choose $0<\tau<T<\infty$. Using the estimate (4.9) in Lemma 4.1 we obtain that $\mathbf{u}_{\lambda}$ is uniformly bounded in $L^{\infty}\left((\tau, T), H^{1}\left(\Gamma_{\infty}\right)\right)$. Moreover each of its components on $\Gamma_{\infty}$, $\left.\partial_{t} \mathbf{u}_{\lambda}\right|_{\Gamma_{\infty}}=\left(\partial_{t} u_{\lambda}^{1}, \ldots, \partial_{t} u_{\lambda}^{N}\right)$ are uniformly bounded in $L^{2}\left((\tau, T), H^{-1}(0, \infty)\right)$. These estimates and Aubin-Lions compactness argument (see for example [42]) imply that each component is relatively compact in $C\left((\tau, T), L_{l o c}^{2}((0, \infty))\right.$ so $\left(\mathbf{u}_{\lambda}\right)_{\lambda>0}$ is relatively compact in $C\left((\tau, T), L_{l o c}^{2}\left(\Gamma_{\infty}\right)\right)$. Cantor's diagonal argument implies that, up to a subsequence, $\left(\mathbf{u}_{\lambda}\right)_{\lambda>0}$ converges toward a function $\mathbf{U}$ in $C\left((0, \infty), L_{l o c}^{2}\left(\Gamma_{\infty}\right)\right)$. In view of estimate (4.8) this convergence implies in particular that for any positive time $t$ the sequence $\mathbf{u}_{\lambda}(t) \rightarrow \mathbf{U}(t)$ in $L^{p}\left(\Gamma_{\infty}\right)$ for any $1<p<\infty$ and the bound in (4.8) transfers to $\mathbf{U}$.

Moreover the above convergence in $C\left((\tau, T), L_{l o c}^{2}\left(\Gamma_{\infty}\right)\right)$ implies that the convergence also holds in $C\left((0, \infty), L_{l o c}^{1}\left(\Gamma_{\infty}\right)\right)$. The uniform tail control (4.11) in Lemma 4.1 shows that the convergence also holds in $C\left((0, \infty), L^{1}\left(\Gamma_{\infty}\right)\right)$. In particular, at time $t=1, \mathbf{u}_{\lambda}(1) \rightarrow \mathbf{U}(1)$ in $L^{1}\left(\Gamma_{\infty}\right)$ and this proves (3.6) when $p=1$. Assume for the moment that we identified the profile $\mathbf{U}$ in $\Gamma_{\infty}$ to be the one given by (3.8), precisely $\mathbf{U}_{M}$. This means that all the family converges to this profile, not only a subsequence. Using that both $\mathbf{u}$ and $\mathbf{U}$ are uniformly bounded in $L^{p}(\Gamma), 1 \leq p<\infty$, with a bound of order $t^{-1 / 2(1-1 / p)}$ and the convergence in $L^{1}\left(\Gamma_{\infty}\right)$ we deduce that (3.6) holds for all $p \in[1, \infty)$. Moreover, since $\mathbf{U}$ have been identified to be $\mathbf{U}_{M}$ explicit computations give us

$$
\left\|\mathbf{U}_{x}(t)\right\|_{L^{2}\left(\Gamma_{\infty}\right)} \leq C(M, N) t^{-3 / 2}, \forall t>0 .
$$

A similar estimates for $\mathbf{u}$ obtained in Theorem 4.2 so to treat the case $p=\infty$ we can argue as follows

$$
\|\mathbf{u}(t)-\mathbf{U}(t)\|_{L^{\infty}\left(\Gamma_{\infty}\right)} \lesssim\left(\left\|\mathbf{u}_{x}(t)\right\|_{L^{2}\left(\Gamma_{\infty}\right)}+\left\|\mathbf{U}_{x}(t)\right\|_{L^{2}\left(\Gamma_{\infty}\right)}\right)^{\frac{1}{2}}\|\mathbf{u}(t)-\mathbf{U}(t)\|_{L^{2}\left(\Gamma_{\infty}\right)}^{\frac{1}{2}}=o\left(t^{-\frac{1}{2}}\right) .
$$

Step II. Identification of the limit. Let us now consider a test function

$$
\varphi: C\left([0, \infty), H^{1}\left(\Gamma_{\infty}\right)\right) \cap C^{1}\left([0, \infty), L^{2}\left(\Gamma_{\infty}\right)\right)
$$

compactly supported in time and such that $\varphi^{e}(t, 0)=\varphi^{e^{\prime}}(t, 0)$ for all $e, e^{\prime} \in \Gamma_{\infty}$ (that is, we ask for continuity at the unique node of $\left.\Gamma_{\infty}\right)$. For each $t \geq 0$, we extend $\varphi(t)$ to the whole $\Gamma^{\lambda}$, to this end we take a function $\widetilde{\varphi}_{\lambda}:[0, \infty) \times \Gamma^{\lambda} \rightarrow \mathbb{R}$ such that $\widetilde{\varphi}_{\lambda}$ is constant in the finite part of the graph $\Gamma_{f}^{\lambda}$, that is,

$$
\widetilde{\varphi}_{\lambda}(t, x)= \begin{cases}\varphi(t, x) & \text { if } x \in \Gamma_{\infty}, \\ \varphi(t, 0) & \text { if } x \in \Gamma_{f}^{\lambda},\end{cases}
$$


It follows that $\widetilde{\varphi} \in C\left([0, \infty), \mathcal{Q}\left(\Gamma^{\lambda}\right)\right.$, i.e. it is not only in $H^{1}\left(\Gamma^{\lambda}\right)$ but it is also continuos. Since $\mathbf{u}_{\lambda} \in C\left([0, \infty), L^{1}(\Gamma) \cap D\left(\Delta_{\Gamma}\right)\right)$ we multiply equation (4.7) by $\widetilde{\varphi}_{\lambda}$ and obtain

$$
\begin{aligned}
0= & \int_{0}^{\infty} \int_{\Gamma_{\infty}} \mathbf{u}_{\lambda} \varphi_{t}-\int_{0}^{\infty} \int_{\Gamma_{\infty}} \mathbf{u}_{\lambda, x} \varphi_{x} \\
& +\int_{0}^{\infty} \int_{\Gamma_{f}^{\lambda}}\left(\mathbf{u}_{\lambda} \widetilde{\varphi}_{\lambda, t}-\mathbf{u}_{\lambda, x} \widetilde{\varphi}_{\lambda, x}\right)+\int_{\Gamma^{\lambda}} \mathbf{u}_{\lambda}(0, x) \widetilde{\varphi}_{\lambda}(0, x) d x \\
= & \int_{0}^{\infty} \int_{\Gamma_{\infty}} \mathbf{u}_{\lambda} \varphi_{t}-\int_{0}^{\infty} \int_{\Gamma_{\infty}} \mathbf{u}_{\lambda, x} \varphi_{x}+\int_{0}^{\infty} \int_{\Gamma_{f}^{\lambda}} \mathbf{u}_{\lambda} \widetilde{\varphi}_{\lambda, t}+\int_{\Gamma^{\lambda}} \mathbf{u}_{\lambda}(0, x) \widetilde{\varphi}_{\lambda}(0, x) d x \\
= & \int_{0}^{\infty} \int_{\Gamma_{\infty}} \mathbf{u}_{\lambda} \varphi_{t}-\int_{0}^{\infty} \int_{\Gamma_{\infty}} \mathbf{u}_{\lambda, x} \varphi_{x}+\int_{0}^{\infty} \varphi_{t}(t, 0) \int_{\Gamma_{f}^{\lambda}} \mathbf{u}_{\lambda}+\int_{\Gamma^{\lambda}} \mathbf{u}_{\lambda}(0, x) \widetilde{\varphi}_{\lambda}(0, x) d x \\
:= & I_{1}^{\lambda}-I_{2}^{\lambda}+I_{3}^{\lambda}+I_{4}^{\lambda},
\end{aligned}
$$

where we used that $\widetilde{\varphi}_{\lambda}$ is constant on $\Gamma_{f}^{\lambda}$.

Using the decay of the $L^{2}$-norm of $\mathbf{u}_{\lambda}$ we obtain that $\mathbf{u}_{\lambda}$ is uniformly bounded in $L^{2}\left((0, T), L^{2}\left(\Gamma_{\infty}\right)\right)$ :

$$
\int_{0}^{T}\left\|\mathbf{u}_{\lambda}(t)\right\|_{L^{2}\left(\Gamma^{\lambda}\right)}^{2} d t \lesssim \int_{0}^{T} \frac{1}{\sqrt{t}} d t \lesssim \sqrt{T} .
$$

This means that up to a subsequence $\mathbf{u}_{\lambda} \rightarrow \mathbf{U}$ in $L^{2}\left((0, T), L^{2}\left(\Gamma_{\infty}\right)\right)$. Using that $\varphi$ has compact support in time we get

$$
I_{1}^{\lambda} \rightarrow \int_{0}^{\infty} \int_{\Gamma_{\infty}} \mathbf{U}(t, x) \varphi_{t}(t, x) d x d t .
$$

For the third term, $I_{3}^{\lambda}$, we use again the decay in the $L^{2}$-norm to obtain

$$
\left|\int_{\Gamma_{f}^{\lambda}} \mathbf{u}_{\lambda}(t, x) d x\right| \leq \sum_{e \in \Gamma_{f}^{\lambda}} \int_{0}^{l_{e} / \lambda}\left|u_{\lambda}^{e}(t, x)\right| d x \leq \sum_{e \in \Gamma_{f}^{\lambda}}\left\|u_{\lambda}^{e}(t)\right\|_{L^{2}(e)}\left(l_{e} / \lambda\right)^{\frac{1}{2}} \leq \frac{C(\Gamma)\left\|\mathbf{u}_{0}\right\|_{L^{1}(\Gamma)}}{\lambda^{\frac{1}{2}} t^{\frac{1}{4}}} .
$$

Integrating in time and using that $\varphi$ has compact support in time we obtain that $I_{3}^{\lambda} \rightarrow 0$ as $\lambda \rightarrow \infty$.

For the last term $I_{4}^{\lambda}$ we split it as follows

$$
\begin{aligned}
I_{4}^{\lambda}= & \int_{\Gamma^{\infty}} \mathbf{u}_{\lambda}(0, x) \widetilde{\varphi}_{\lambda}(0, x) d x+\int_{\Gamma_{f}^{\lambda}} \mathbf{u}_{\lambda}(0, x) \widetilde{\varphi}_{\lambda}(0, x) d x \\
= & \int_{\Gamma^{\infty}} \mathbf{u}_{\lambda}(0, x) \varphi(0, x) d x+\int_{\Gamma_{f}^{\lambda}} \mathbf{u}_{\lambda}(0, x) \varphi(0,0) d x \\
= & \varphi(0,0) \int_{\Gamma^{\lambda}} \mathbf{u}_{\lambda}(0, x) d x+\int_{\Gamma^{\infty}} \mathbf{u}_{\lambda}(0, x)(\varphi(0, x)-\varphi(0,0)) d x \\
= & \varphi(0,0) \int_{\Gamma} \mathbf{u}_{0}(x) d x+\int_{\Gamma^{\infty}} \mathbf{u}_{0}(x)\left(\varphi\left(0, \frac{x}{\lambda}\right)-\varphi(0,0)\right) d x \\
& \rightarrow \varphi(0,0) \int_{\Gamma} \mathbf{u}_{0}(x) d x, \quad \text { as } \lambda \rightarrow \infty .
\end{aligned}
$$


It remains to prove that

$$
I_{2}^{\lambda} \rightarrow \int_{0}^{\infty} \int_{\Gamma^{\infty}} \mathbf{U}_{x}(t, x) \varphi_{x}(t, x) d x d t
$$

First, we prove that for any $\tau>0$ the integrals in $(0, \tau)$ are small independently on $\lambda$. Indeed, using (4.9) we have

$$
\left|\int_{0}^{\tau} \int_{\Gamma_{\infty}} \mathbf{u}_{\lambda, x}(t, x) \varphi_{x}(t, x) d x d t\right| \leq\left\|\mathbf{u}_{\lambda, x}\right\|_{L^{1}\left((0, \tau), L^{2}\left(\Gamma_{\infty}\right)\right)}\left\|\varphi_{x}\right\|_{L^{\infty}\left((0, \tau), L^{2}\left(\Gamma_{\infty}\right)\right)} \lesssim \tau^{\frac{1}{4}}
$$

We recall that at the begining of Step I we proved that $\mathbf{u}_{\lambda}(t) \rightarrow \mathbf{U}(t)$ in $L^{p}\left(\Gamma_{\infty}\right)$ for any $1<$ $p<\infty$. Moreover, $\left(\mathbf{u}_{\lambda, x}(t)\right)_{\lambda>0}$ is uniformly bounded in $L^{2}\left(\Gamma_{\infty}\right)$. Hence, up to a subsequence $\mathbf{u}_{\lambda, x}(t) \rightarrow \mathbf{U}_{x}(t)$ in $L^{2}\left(\Gamma_{\infty}\right)$. Estimate (4.9) transfers to $\mathbf{U}$ so $\left\|\mathbf{U}_{x}(t)\right\|_{L^{2}\left(\Gamma_{\infty}\right)} \lesssim t^{-\frac{3}{4}}$ for all $t>0$ and a similar estimate holds for $\mathbf{U}$

$$
\left|\int_{0}^{\tau} \int_{\Gamma_{\infty}} \mathbf{U}_{x} \varphi_{x} d x\right| \lesssim \tau^{\frac{1}{4}}
$$

In view of (4.10) we get

$$
\int_{\tau}^{\infty} \int_{\Gamma_{\infty}} \mathbf{u}_{\lambda, x}(t, x) \varphi_{x}(t, x) d x d t \rightarrow \int_{\tau}^{\infty} \int_{\Gamma_{\infty}} \mathbf{U}_{x}(t, x) \varphi_{x}(t, x) d x d t
$$

which proves the desired limit for $I_{2}^{\lambda}$.

In view of the above results it follows that the limit point $\mathbf{U}$ satisfies

$$
\int_{0}^{\infty} \int_{\Gamma_{\infty}}\left(\mathbf{U}(t, x) \varphi_{t}(t, x)-\mathbf{U}_{x}(t, x) \varphi_{x}(t, x)\right) d x d t+M \varphi(0,0)=0 .
$$

From Step I we know that the limit point $\mathbf{U}$ belongs to $C\left((0, \infty), L^{1}\left(\Gamma_{\infty}\right)\right)$ and moreover $\|\mathbf{U}(t)\|_{L^{1}\left(\Gamma_{\infty}\right)} \leq\left\|\mathbf{u}_{0}\right\|_{L^{1}\left(\Gamma_{\infty}\right)}$. Also the energy estimate (4.10) shows that $\mathbf{u}_{\lambda}$ is uniformly bounded in $L^{2}\left((\tau, T), D\left(\mathcal{Q}_{\Gamma_{\infty}}\right)\right)$ hence the limit point $\mathbf{U}$ belongs to $L^{2}\left((\tau, T), D\left(\mathcal{Q}_{\Gamma_{\infty}}\right)\right)$ for any $\tau>0$. This implies that $\mathbf{U}(t) \in D\left(\mathcal{Q}_{\Gamma_{\infty}}\right)$ for a.e. $t>0$.

The continuity of $\mathbf{U}$ at the common vertex of $\Gamma_{\infty}$ (or the Neumann boundary condition if $\Gamma_{\infty}$ has only one edge) guarantees that for a test function $\varphi$ which is more regular, $\varphi \in$ $C\left([0, \infty), D\left(\Delta_{\Gamma_{\infty}}\right)\right) \cap C^{1}\left([0, \infty), L^{2}\left(\Gamma_{\infty}\right)\right)$ and compactly supported in time, we can perform one more integration by parts and obtain that $\mathbf{U}$ satisfies

$$
\int_{0}^{\infty} \int_{\Gamma_{\infty}} \mathbf{U}(t, x)\left(\varphi_{t}(t, x)+\varphi_{x x}(t, x)\right) d x d t+M \varphi(0,0)=0
$$

For the above equation we need only $\mathbf{U} \in L_{l o c}^{1}\left((0, \infty), L^{1}\left(\Gamma_{\infty}\right)\right)$ in order to apply the arguments in [6] and prove the uniqueness of the profile $\mathbf{U}$ satisfying (4.12).

An easy computation shows that $\mathbf{U}_{M}=\left(U^{1}, \ldots, U^{N}\right)$ given by

where

$$
U_{M}^{k}(t, x)=\frac{2 M}{N} G_{t}(x), \quad k=1, \ldots, N
$$

$$
G_{t}(x)=\frac{1}{\sqrt{4 \pi t}} e^{-\frac{x^{2}}{4 t}}
$$


is the classical heat kernel, verifies identity (4.12). Assume that we have two solutions $\mathbf{U}_{1}$ and $\mathbf{U}_{2}$ of problem (4.12) in $L_{l o c}^{1}\left((0, \infty), L^{1}\left(\Gamma_{\infty}\right)\right)$. Then $\mathbf{v}=\mathbf{U}_{1}-\mathbf{U}_{2} \in L_{l o c}^{1}\left((0, \infty), L^{1}\left(\Gamma_{\infty}\right)\right)$ satisfies

$$
\int_{0}^{\infty} \int_{\Gamma_{\infty}} \mathbf{v}\left(\varphi_{t}+\varphi_{x x}\right)=0
$$

for all functions $\varphi \in C\left([0, \infty), D\left(\Delta_{\Gamma_{\infty}}\right)\right) \cap C^{1}\left([0, \infty), L^{2}\left(\Gamma_{\infty}\right)\right)$. We show what all the components $v_{1}, \ldots, v_{N}$ of $\mathbf{v}$ are equal and vanish identically. Let us fix a $T>0$ and a function $\psi \in$ $C^{1,2}([0, T] \times[0, \infty))$ with $\psi(T) \equiv 0, \psi(0, t)=0$ for $t \in[0, T]$. Choosing $\varphi=(\psi,-\psi, 0, \ldots, 0)$ we obtain that

$$
\int_{0}^{\infty} \int_{\Gamma_{\infty}}\left(v_{1}-v_{2}\right)\left(\psi_{t}+\psi_{x x}\right)=0
$$

Using [6, Lemma 3] we obtain that $v_{1} \equiv v_{2}$ in $[0, T]$. Similarly all the components of $\mathbf{v}$ are identical. Let us now choose a similar $\psi$ but now assume that $\psi_{x}(t, 0)=0$ instead of the Dirichlet boundary condition at $x=0$. Choosing $\varphi=(\psi, \ldots, \psi)$ in (4.13) and using that all the components are identical we obtain

$$
\int_{0}^{T} \int_{0}^{\infty} v_{1}\left(\psi_{t}+\psi_{x x}\right)=0 .
$$

For any $f \in L^{2}\left((0, T), L^{2}(0, \infty)\right)$ we solve the backward heat equation (notice that here the "initial condition" is taken at $t=T$ and time is reversed)

$$
\begin{cases}\psi_{t}+\psi_{x x}=f, & x \in(0, \infty), t \in(0, T), \\ \psi(T, x)=0, & x \in(0, \infty), \\ \psi_{x}(t, 0)=0, & t \in(0, T),\end{cases}
$$

and obtain a function that $\psi$ that can be used as test function in the integral identity satisfied by $v_{1}$. This shows that $v_{1} \equiv 0$ in $(0, T) \times(0, \infty)$ so $\mathbf{v} \equiv 0$ on $(0, T) \times \Gamma_{\infty}$. We conclude that every limit point of $\mathbf{u}_{\lambda}, \mathbf{U}$, is given by (4.12) and therefore the results in Step I hold for the whole family $\left(u_{\lambda}\right)$ and not only for a subsequence.

\section{Step III. Convergence on the compact part of the graph.}

Let us consider $\mathbf{w}(t) \in D\left(\mathcal{Q}_{\Gamma_{\infty}}\right)$, defined as follows

$$
\mathbf{w}(t)=\mathbf{u}(t)-\mathbf{U}_{M}(t) .
$$

Since the graph $\Gamma_{f}$ is finite it is sufficient to prove (3.7) in the case $p=\infty$. Let us recall that since $\Gamma$ has at least one infinite edge we have for any $v \in D\left(\mathcal{Q}_{\Gamma_{\infty}}\right)$ that

$$
\|v\|_{L^{\infty}(\Gamma)} \leq C(\Gamma)\left\|v_{x}\right\|_{L^{2}(\Gamma)}^{\frac{1}{2}}\|v\|_{L^{2}(\Gamma)}^{\frac{1}{2}} .
$$

We apply the above inequality to $\mathbf{w}$

$$
\left.\|\mathbf{w}(t)\|_{L^{\infty}\left(\Gamma_{f}\right)} \leq\|\mathbf{w}(t)\|_{L^{\infty}(\Gamma)} \leq C(\Gamma)\right)\left\|\mathbf{w}_{x}\right\|_{L^{2}(\Gamma)}^{\frac{1}{2}}\|\mathbf{w}(t)\|_{L^{2}(\Gamma)}^{\frac{1}{2}} .
$$

Observe that $\mathbf{w}_{x}(t)=\mathbf{u}_{x}(t)-\mathbf{U}_{M, x}(t, x) 1_{\Gamma_{\infty}}(x)$. Explicit computations on $\mathbf{U}_{M}$ and (4.5) gives us

$$
\left\|\mathbf{w}_{x}(t)\right\|_{L^{2}(\Gamma)} \leq\left\|\mathbf{u}_{x}(t)\right\|_{L^{2}(\Gamma)}+\left\|\mathbf{U}_{M, x}(t)\right\|_{L^{2}\left(\Gamma_{\infty}\right)} \leq C(\Gamma)\left(\left\|\mathbf{u}_{0}\right\|_{L^{1}(\Gamma)}+M\right) t^{-3 / 4} .
$$


It is then sufficient to prove that $\|\mathbf{w}(t)\|_{L^{2}(\Gamma)}=o\left(t^{-\frac{1}{4}}\right)$ as $t \rightarrow \infty$. This estimate on $\Gamma_{\infty}$ has been proved in Step I. For $\Gamma_{f}$ we use (4.4) for $p=\infty$ and the explicit form of $\mathbf{u}_{M}$ on $\Gamma_{f}$ :

$$
\begin{aligned}
\|\mathbf{w}(t)\|_{L^{2}\left(\Gamma_{f}\right)} & \leq C(\Gamma)\|\mathbf{w}(t)\|_{L^{\infty}\left(\Gamma_{f}\right)} \leq C(\Gamma)\left(\|\mathbf{u}(t)\|_{L^{\infty}\left(\Gamma_{f}\right)}+\left\|\mathbf{U}_{M}(t)\right\|_{L^{\infty}\left(\Gamma_{f}\right)}\right) \\
& \leq C(\Gamma) t^{-\frac{1}{2}}=o\left(t^{-\frac{1}{4}}\right), t \rightarrow \infty .
\end{aligned}
$$

This complete the case of the finite graph and finishes the proof.

\section{THE NONLOCAL PROBLEM}

In this section we consider the nonlocal problem in the metric graph $\Gamma$,

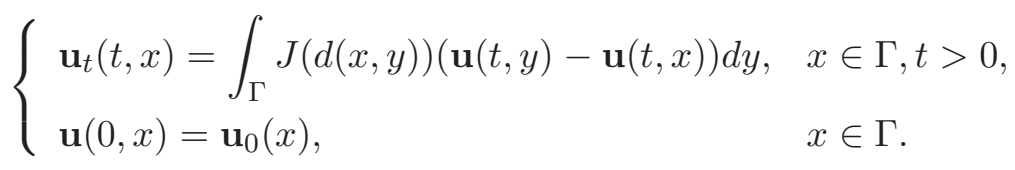

Here $d(x, y)$ stands for the distance between two points in the graph. As we mentioned in the introduction, this distance $d(x, y)$ is the length of the minimal path that joins $x$ and $y$. The kernel $J \in L^{1}(\mathbb{R}) \cap L^{1}\left(\mathbb{R},|x|^{2}\right)$ is assumed to be symmetric, $J(z)$ is non-increasing for $z>0$, $J$ is nonnegative in $\mathbb{R}$ and positive in neighbourhood of the origin.

Our first goal is to show existence and uniqueness of solution for the problem (5.1).

Theorem 5.1. For any $\mathbf{u}_{0} \in L^{p}(\Gamma), 1 \leq p \leq \infty$, there exists an unique solution $\mathbf{u} \in$ $C\left([0, \infty), L^{p}(\Gamma)\right)$ of system (5.1) satisfying

$$
\|\mathbf{u}(t)\|_{L^{p}(\Gamma)} \leq\left\|\mathbf{u}_{0}\right\|_{L^{p}(\Gamma)} .
$$

Also, for nonnegative initial datum the solution remains nonnegative.

For any $\mathbf{u}_{0} \in L^{2}(\Gamma)$ the following energy estimate holds

$$
\int_{\Gamma} \mathbf{u}^{2}(t, x) d x+\int_{0}^{t} \int_{\Gamma} \int_{\Gamma} J(d(x, y))(\mathbf{u}(s, x)-\mathbf{u}(s, y))^{2} d x d y d s=\int_{\Gamma} \mathbf{u}_{0}^{2}(x) d x .
$$

Moreover,

$$
\mathcal{E}_{\Gamma}^{J}(\mathbf{u}(t), \mathbf{u}(t)):=\int_{\Gamma} \int_{\Gamma} J(d(x, y))(\mathbf{u}(t, x)-\mathbf{u}(t, y))^{2} d x d y \leq \frac{\left\|\mathbf{u}_{0}\right\|_{L^{2}(\Gamma)}^{2}}{t} .
$$

Proof of Theorem 5.1. The existence and uniqueness follow easily since the operator

$$
\mathbf{u} \mapsto L(\mathbf{u}):=\int_{\Gamma} J(d(x, y))(\mathbf{u}(y)-\mathbf{u}(x)) d y
$$

is bounded between any $L^{p}(\Gamma)$ spaces (we refer to [41] for extra details). Therefore, problem (5.1) has a unique strong solution $\mathbf{u} \in C^{\infty}(\mathbb{R}, X)$, for any $X=L^{p}(\Gamma)$ given by $\mathbf{u}(t)=e^{L t} \mathbf{u}_{0}$. The mapping $t \in(0, \infty) \mapsto \mathbf{u}(t)=e^{L t} \mathbf{u}_{0} \in X$ is analytic. Moreover, the mapping $\left(t, \mathbf{u}_{0}\right) \mapsto$ $e^{L t} \mathbf{u}_{0}$ is continuous and contractive, that is, $\|\mathbf{u}(t)\|_{L^{p}(\Gamma)} \leq\left\|\mathbf{u}_{0}\right\|_{L^{p}(\Gamma)}$ holds. Indeed, for any function $\rho \in C^{1}(\mathbb{R})$ with $\rho^{\prime}$ nondecreasing we have

$$
\frac{d}{d t} \int_{\Gamma} \rho(\mathbf{u}(t, x)) d x=\left(\mathbf{u}_{t}(t), \rho^{\prime}(\mathbf{u}(t))\right)=\left(L \mathbf{u}(t), \rho^{\prime}(\mathbf{u}(t))\right)=-\mathcal{E}_{\Gamma}^{J}\left(\mathbf{u}(t), \rho^{\prime}(\mathbf{u}(t))\right) \leq 0
$$


An approximation argument shows that for any convex function $\rho$ the map $t \rightarrow \int_{\Gamma} \rho(\mathbf{u}(t, x)) d x$ is nonincreasing. Particular cases $\rho(s)=|s|^{p}, 1 \leq p<\infty$ show the contractivity in the $L^{p_{-}}$ norms. When $p=\infty$ we consider $\rho(s)=(|s|-M)^{+}$and $M=\left\|\mathbf{u}_{0}\right\|_{L^{\infty}(\Gamma)}$. For $\rho(s)=s^{+}$we obtain the positivity property of the semigroup.

In particular we have that

$$
\frac{1}{2} \frac{d}{d t} \int_{\Gamma} \mathbf{u}^{2}(t, x) d x=-\frac{1}{2} \int_{\Gamma} \int_{\Gamma} J(d(x, y))(\mathbf{u}(t, x)-\mathbf{u}(t, y))^{2} d x d y .
$$

Estimate (5.2) is classical for any self-adjoint operator $L$ satisfying $(L u, u) \leq 0$, see for example [13, Th.3.2.1] which finishes the proof.

5.1. Relaxation limit. Let us now consider the following relaxation problem: for each $\varepsilon>0$ consider the system

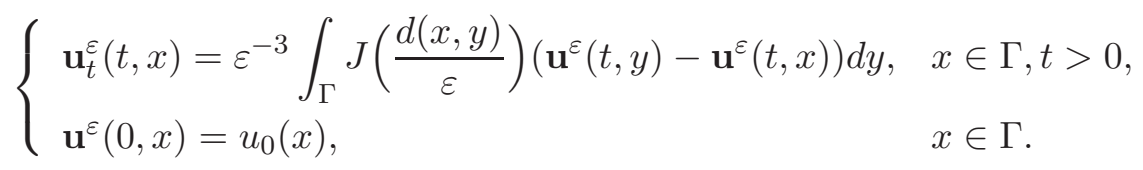

and analyze the limit problem when $\varepsilon \rightarrow 0$. Let us mention that here we fix the initial datum in contrast with the analysis of the first term in the asymptotic behavior of the solutions by self-similarity where we also have to rescale the initial data. Here we use compactness arguments instead of scaling ones (the kernel of the nonlocal operator is rescaled with $\varepsilon$, but the spatial domain $\Gamma$ in which solutions are defined is unchanged). Recall that we assumed

$$
\frac{1}{2} \int_{\mathbb{R}} z^{2} J(z) d z=1
$$

Proof of Theorem 3.2. Let us remark that we have the following energy estimate

$$
\int_{\Gamma}\left(\mathbf{u}^{\varepsilon}\right)^{2}(t, x) d x+\varepsilon^{-3} \int_{0}^{T} \int_{\Gamma} \int_{\Gamma} J\left(\frac{d(x, y)}{\varepsilon}\right)\left(\mathbf{u}^{\varepsilon}(t, y)-\mathbf{u}^{\varepsilon}(t, x)\right)^{2} d x d y d t=\int_{\Gamma} \mathbf{u}_{0}^{2}(x) d x .
$$

We will use the above identity in three different ways, by taking the same edge, two adjacent edges or two edges that does not have a vertex in common.

As before, we divide our arguments into several steps.

Step I. We observe that $\mathbf{u}^{\varepsilon}$ is uniformly bounded in $C\left([0, T], L^{2}(\Gamma)\right)$, so in particular in $L^{2}\left((0, T), L^{2}(\Gamma)\right)$. So there exists $\mathbf{U}=\left(U_{e}\right)_{e \in E} \in L^{2}\left((0, T), L^{2}(\Gamma)\right)$ such that, up to a subsequence, $\mathbf{u}^{\varepsilon} \rightarrow \mathbf{U}$ in $L^{2}\left((0, T), L^{2}(\Gamma)\right)$. In particular $u_{e}^{\varepsilon} \rightarrow U_{e}$ in $L^{2}\left((0, T), L^{2}(e)\right)$ for any any edge $e \in E$.

Let us consider an arbitrary edge $e$ parametrized by $[0, l]$ or $[0, \infty)$. In both cases by Lemma 6.2 the estimate above guarantees that for any $T>0$

$$
\varepsilon^{-3} \int_{0}^{T} \int_{e} \int_{e} J\left(\frac{d(x, y)}{\varepsilon}\right)\left(u^{\varepsilon}(t, y)-u^{\varepsilon}(t, x)\right)^{2} d x d y d t \leq \int_{\Gamma} u_{0}^{2}(x) d x
$$

and then $\mathbf{U} \in L^{2}\left((0, T), H^{1}(e)\right)$ such that

$$
u^{\varepsilon} \rightarrow U^{e} \text { in } L^{2}\left((0, T), L_{l o c}^{2}(e)\right) .
$$


This shows that $\mathbf{U} \in L^{2}\left((0, T), H^{1}(\Gamma)\right)$. Moreover, in view of the results in Lemma 6.2, for any $\varphi \in L^{2}\left((0, T), H^{1}(e)\right)$ we have

$$
\begin{gathered}
\varepsilon^{-3} \int_{0}^{T} \int_{e} \int_{e} J\left(\frac{d(x, y)}{\varepsilon}\right)\left(u^{\varepsilon}(t, y)-u^{\varepsilon}(t, x)\right)(\varphi(t, y)-\varphi(t, x)) d x d y d t \\
\rightarrow A(J) \int_{0}^{T} \int_{e} U_{x}(t, x) \varphi_{x}(t, x) d x d t, \quad \text { as } \varepsilon \rightarrow 0,
\end{gathered}
$$

where $A(J)=\int_{\mathbb{R}} J(z) z^{2} d z$.

Step II. We now show that $\mathbf{U} \in L^{2}\left((0, T), D\left(\mathcal{Q}_{\Gamma}\right)\right)$, i.e. it belongs to $L^{2}\left((0, T), H^{1}(e)\right)$ and $\mathbf{U}(t)$ is also continuous at any vertex for a.e. $t>0$.

Let us now consider two edges $e$ and $e^{\prime}$ that have a common vertex $v$. We first prove that the limit function $\mathbf{U}=\left(U_{e}\right)_{e}$ is continuous at any internal vertex, i.e. $U_{e}(t, v)=U_{e^{\prime}}(t, v)$, for a.e. $t$. Let us assume that the two edges are parametrized by $I \subset(-\infty, 0]$ and $I^{\prime} \subset[0, \infty)$. In view of energy estimate (5.4) it follows that the function $w^{\varepsilon}$ defined by

$$
w^{\varepsilon}(t, x)= \begin{cases}u_{e}^{\varepsilon}(t, x), & x \in I \\ u_{e^{\prime}}^{\varepsilon}(t, x), & x \in I^{\prime}\end{cases}
$$

satisfies

$$
\varepsilon^{-3} \int_{0}^{T} \int_{I \cup I^{\prime}} \int_{I \cup I^{\prime}} J\left(\frac{x-y}{\varepsilon}\right)\left(w^{\varepsilon}(t, y)-w^{\varepsilon}(t, x)\right)^{2} d x d y d t \leq\left\|u_{0}\right\|_{L^{2}(\Gamma)}^{2} .
$$

Thus $w^{\varepsilon}$ converges, up to a subsequence, weakly to a function $w \in L^{2}\left((0, T), H^{1}\left(I \cup I^{\prime}\right)\right)$. From Step I we know that $u_{e}^{\varepsilon}$ converges to $u_{e}, u_{e^{\prime}}^{\varepsilon}$ converges to $u_{e^{\prime}}$ where the function obtained from the pair $u_{e}, u_{e^{\prime}}$ belongs to $L^{2}\left((0, T), H^{1}\left(I \cup I^{\prime}\right)\right)$. Hence, it follows that

$$
u_{e}(t, 0-)=u_{e^{\prime}}(t, 0+)
$$

for a.e. $t>0$. Thanks to this property the limit $\mathbf{U}$ belongs to $L^{2}\left((0, T), D\left(\mathcal{Q}_{\Gamma}\right)\right)$ for any $T>0$. Moreover, by Lemma 6.3, for any $\varphi \in L^{2}\left((0, T), D\left(\mathcal{Q}_{\Gamma}\right)\right)$, we have

$$
\varepsilon^{-3} \int_{0}^{T} \int_{e} \int_{e^{\prime}} J\left(\frac{d(x, y)}{\varepsilon}\right)(\varphi(t, y)-\varphi(t, x))^{2} d x d y d t \rightarrow 0
$$

and then we get

$$
\varepsilon^{-3} \int_{0}^{T} \int_{e} \int_{e^{\prime}} J\left(\frac{d(x, y)}{\varepsilon}\right)\left(u^{\varepsilon}(t, y)-u^{\varepsilon}(t, x)\right)(\varphi(t, y)-\varphi(t, x)) d x d y d t \rightarrow 0 .
$$

Step III. Let us consider two edges $e$ and $e^{\prime}$ which do not have a common endpoint. In this case we will prove that for any function $\varphi \in L^{2}\left((0, T), L^{2}\left(e \cup e^{\prime}\right)\right)$

$$
\varepsilon^{-3} \int_{0}^{T} \int_{e} \int_{e^{\prime}} J\left(\frac{d(x, y)}{\varepsilon}\right)(\varphi(t, y)-\varphi(t, x))^{2} d x d y d t \rightarrow 0, \text { as } \varepsilon \rightarrow 0 .
$$

As a consequence using (5.4) we obtain

$$
\varepsilon^{-3} \int_{0}^{T} \int_{e} \int_{e^{\prime}} J\left(\frac{d(x, y)}{\varepsilon}\right)\left(u^{\varepsilon}(t, y)-u^{\varepsilon}(t, x)\right)(\varphi(t, y)-\varphi(t, x)) d x d y d t \rightarrow 0, \text { as } \varepsilon \rightarrow 0 .
$$


Let us now prove the first limit. Indeed, we have

$$
\begin{aligned}
\varepsilon^{-3} \int_{0}^{T} & \int_{e} \int_{e^{\prime}} J\left(\frac{d(x, y)}{\varepsilon}\right)(\varphi(t, y)-\varphi(t, x))^{2} d x d y d t \\
& \leq 2 \varepsilon^{-3} \int_{0}^{T} \int_{e^{\prime}} \varphi^{2}(t, y) \int_{e} J\left(\frac{d(x, y)}{\varepsilon}\right) d x d y d t+2 \varepsilon^{-3} \int_{0}^{T} \int_{e} \varphi^{2}(t, x) \int_{e^{\prime}} J\left(\frac{d(x, y)}{\varepsilon}\right) d y d x d t .
\end{aligned}
$$

By symmetry it is sufficient to consider only the first term in the right hand side. Assume that the distance in the graph between the two edges is $\alpha>0$ and that $e$ is parametrized by $\left(0, l_{e}\right)$. Then $d(x, y) \geq x+\alpha$ for any $y \in e^{\prime}$ and since $J$ is a non-increasing function we have

$$
\varepsilon^{-3} \int_{e} J\left(\frac{d(x, y)}{\varepsilon}\right) d x \leq \varepsilon^{-3} \int_{0}^{l_{e}} J\left(\frac{x+\alpha}{\varepsilon}\right) d x=\varepsilon^{-2} \int_{\alpha / \varepsilon}^{l_{e} / \varepsilon} J(z) d z \leq \frac{1}{\alpha^{2}} \int_{\alpha / \varepsilon}^{l_{e} / \varepsilon} J(z) z^{2} d z .
$$

Using that $J$ has a finite second momentum and that $\varphi \in L^{2}\left((0, T), L^{2}(e)\right)$ we obtain that the considered term tends to zero as $\varepsilon \rightarrow 0$ which proves that (5.5) holds.

Step IV. We prove that $\mathbf{U} \in C\left([0, T], L^{2}(\Gamma)\right)$. In view of Step II it is sufficient to show that $\mathbf{U}_{t} \in L^{2}\left((0, T), D\left(\mathcal{Q}_{\Gamma}\right)^{\prime}\right)$. We show that $\mathbf{U}_{t}^{\varepsilon}$ is uniformly bounded in $L^{2}\left((0, T), D\left(\mathcal{Q}_{\Gamma}\right)^{\prime}\right)$ so in the limit we obtain the desired property for $\mathbf{U}$.

Let us take a function $\varphi \in L^{2}\left((0, T), D\left(\mathcal{Q}_{\Gamma}\right)\right)$. Using identity (5.4), it follows that

$$
\begin{aligned}
\left|\int_{0}^{T} \int_{\Gamma} \mathbf{U}_{t}^{\varepsilon} \varphi\right|^{2} & =\mid \frac{\varepsilon^{-3}}{2} \int_{0}^{T} \int_{\Gamma} \int_{\Gamma} J\left(\frac{d(x, y)}{\varepsilon}\right)\left(\mathbf{U}^{e}(t, y)-\mathbf{U}^{e}(t, x)\right)\left(\varphi(t, y)-\left.\varphi(t, x) d x d y d t\right|^{2}\right. \\
& \leq\left\|\mathbf{u}_{0}\right\|_{L^{2}(\Gamma)}^{2} \frac{\varepsilon^{-3}}{4} \int_{0}^{T} \int_{\Gamma} \int_{\Gamma} J\left(\frac{d(x, y)}{\varepsilon}\right)(\varphi(t, y)-\varphi(t, x))^{2} d x d y d t .
\end{aligned}
$$

It is sufficient to show that the following holds for any $\psi \in D\left(\mathcal{Q}_{\Gamma}\right)$ and $\varepsilon>0$ :

$$
\varepsilon^{-3} \int_{\Gamma} \int_{\Gamma} J\left(\frac{d(x, y)}{\varepsilon}\right)(\psi(y)-\psi(x))^{2} d x d y \leq C(\Gamma, J) \int_{\Gamma}\left(\psi_{x}^{2}+\psi^{2}\right) d x .
$$

To prove that we split the integral in the left hand side in integrals over adjacent edges or not. Let us take two edges $e$ and $e^{\prime}$ having no common point. In view of Step III

$$
\varepsilon^{-3} \int_{e} \int_{e^{\prime}} J\left(\frac{d(x, y)}{\varepsilon}\right)(\psi(y)-\psi(x))^{2} d x d y \leq C(\Gamma, J) \int_{e \cup e^{\prime}} \psi^{2}(x) d x .
$$

When two edges $e$ and $e^{\prime}$ have a common endpoint we can parametrize them as in Step II and then $d(x, y)=|x-y|, \psi \in H^{1}\left(I \cup I^{\prime}\right)$ and we can use the real line case (see for example [5. Th. 1])

$$
\begin{aligned}
\varepsilon^{-3} \int_{e \cup e^{\prime}} \int_{e \cup e^{\prime}} J\left(\frac{d(x, y)}{\varepsilon}\right)(\psi(y)-\psi(x))^{2} d x d y & =\varepsilon^{-3} \int_{I \cup I^{\prime}} \int_{I \cup I^{\prime}} J\left(\frac{|x-y|}{\varepsilon}\right)(\psi(y)-\psi(x))^{2} d x d y \\
& \leq C\left(I, I^{\prime}, J\right) \int_{I \cup I^{\prime}}\left(\psi^{2}+\psi_{x}^{2}\right) d x .
\end{aligned}
$$

Hence (5.6) holds and as a consequence

$$
\left\|\mathbf{U}_{t}^{\varepsilon}\right\|_{L^{2}\left((0, T), D\left(\mathcal{Q}_{\Gamma}\right)^{\prime}\right)} \leq C(\Gamma, J)\left\|\mathbf{u}_{0}\right\|_{L^{2}(\Gamma)} .
$$


Step V. Let us now consider $\mathbf{u}^{\varepsilon} \in C\left([0, \infty), L^{2}(\Gamma)\right)$ solution of problem (5.3). We multiply equation (5.3) by a function $\varphi \in C_{c}\left([0, \infty), D\left(\mathcal{Q}_{\Gamma}\right)\right), \varphi_{t} \in C_{c}\left([0, \infty), L^{2}\right)$. It follows that,

$$
\begin{array}{r}
\int_{0}^{\infty} \int_{\Gamma} \mathbf{u}^{\varepsilon} \varphi_{t} d x d t+\frac{\varepsilon^{-3}}{2} \int_{0}^{\infty} \int_{\Gamma} \int_{\Gamma} J\left(\frac{d(x, y)}{\varepsilon}\right)\left(\mathbf{u}^{\varepsilon}(t, y)-\mathbf{u}^{\varepsilon}(t, x)\right)(\varphi(t, y)-\varphi(t, x)) d x d y d t \\
+\int_{\Gamma} \mathbf{u}_{0}(x) \varphi(0, x) d x=0 .
\end{array}
$$

Since $\mathbf{u}^{\epsilon} \rightarrow \mathbf{U}$ in $L^{2}\left((0, \infty), L^{2}(\Gamma)\right)$ we have

$$
\int_{0}^{\infty} \int_{\Gamma} \mathbf{u}^{\varepsilon} \varphi_{t} d x d t \rightarrow \int_{0}^{\infty} \int_{\Gamma} \mathbf{U} \varphi_{t} d x d t
$$

In view of Step I and Step III we get under the assumption (3.10)

$$
\begin{aligned}
\frac{\varepsilon^{-3}}{2} \int_{0}^{\infty} \int_{\Gamma} \int_{\Gamma} J\left(\frac{d(x, y)}{\varepsilon}\right)\left(\mathbf{u}^{\varepsilon}(t, y)-\mathbf{u}^{\varepsilon}(t, x)\right) & (\varphi(t, y)-\varphi(t, x)) d x d y d t \\
& \rightarrow \int_{0}^{\infty} \int_{\Gamma} \mathbf{U}_{x}(t, x) \varphi_{x}(t, x) d x d t
\end{aligned}
$$

Hence the limit point $\mathbf{U} \in C\left([0, \infty), L^{2}(\Gamma)\right) \cap L_{l o c}^{2}\left((0, \infty), D\left(\mathcal{Q}_{\Gamma}\right)\right)$ satisfies

$$
\int_{0}^{\infty} \int_{\Gamma} \mathbf{U}(t, x) \varphi_{t}(t, x) d x d t-\int_{0}^{\infty} \int_{\Gamma} \mathbf{U}_{x}(t, x) \varphi_{x}(t, x) d x d t+\int_{\Gamma} \mathbf{u}_{0}(x) \varphi(0, x) d x=0 .
$$

Classical arguments for the classical heat equation shows that $\mathbf{U}$ is the unique solution to the heat equation in $\Gamma$ with initial datum $\mathbf{u}_{0} \in L^{2}(\Gamma)$.

The proof is now complete.

5.2. Asymptotic behaviour for the nonlocal evolution problem. Now our goal is to analyze the behaviour of solutions to the nonlocal problem.

Before entering into the statements and proofs of our main results let us prove two auxiliary results that will be needed in order to obtain decay bounds for the solutions and the asymptotic behaviour. For the first one we follow ideas from [29] but adapted to the graphs having some infinite edges.

5.2.1. Preliminaries. We now give a decomposition similar to the one done in [29] but on half line intervals. We assume that function $J$ belongs to $L^{1}(\mathbb{R})$ and it is positive in a neighborhood of the origin. To simplify the presentation we introduce the bilinear form

$$
E_{I}^{J}(u, v)=\int_{I} \int_{I} J(x-y)(u(x)-u(y))(v(x)-v(y)) d x d y .
$$

Lemma 5.1. Let $I$ be the half line $(0, \infty)$ or the real line $\mathbb{R}$. For any $u \in L^{2}(I)$ there exists a decomposition $u=v+w$ such that

$$
\left\|v_{x}\right\|_{L^{2}(I)}^{2}+\|w\|_{L^{2}(I)}^{2} \leq C(J) E_{I}^{J}(u, u)
$$

and for any $a>0$ their norms satisfy

$$
\|v\|_{L^{p}(0, a)}+\|w\|_{L^{p}(0, a)} \leq C(J)\|u\|_{L^{p}(0, a+1)}, \quad \forall 1 \leq p \leq \infty .
$$


Proof. The case when $I$ is the whole real line has been proved in [29, Th. 2.1]. We now consider the case $I=(0, \infty)$.

Since $J$ is positive near the origin we can choose a smooth nonnegative function $\rho$ to be supported in $(-1,0) \cap \operatorname{supp} J$, with $\int_{-1}^{0} \rho=1$ and satisfying

$$
|\rho(z)|+\left|\rho^{\prime}(z)\right| \leq c(J)|J(z)|, \quad z \in \mathbb{R} .
$$

In particular this implies that

$$
\|\rho\|_{L^{1}(\mathbb{R})}+\left\|\rho^{\prime}\right\|_{L^{1}(\mathbb{R})} \leq C(J) .
$$

For any $x>0$ we set

$$
v(x)=\int_{0}^{\infty} \rho(x-y) u(y) d y=\int_{x}^{x+1} \rho(x-y) u(y) d y .
$$

Using Hölder inequality integrating with respect to the measure $\rho(x-y) d y$ we immediately obtain that

$$
\|v\|_{L^{p}((0, a))}^{p} \leq\|\rho\|_{L^{1}(\mathbb{R})} \int_{0}^{a} \int_{x}^{x+1}\left|\rho(x-y)\left\|\left.u(y)\right|^{p} d y d x \leq C(J)\right\| u \|_{L^{p}(0, a+1)}^{p} .\right.
$$

Letting $w=u-v$ we obtain the last property.

We now prove that this decomposition satisfies the first property. Observe that since $\rho$ is compactly supported in $(-1,0)$ we have for any $x>0$ that

$$
\int_{0}^{\infty} \rho^{\prime}(x-y) d y=\int_{-\infty}^{x} \rho^{\prime}(z) d z=\rho(x)=0 .
$$

Thus

and

$$
v_{x}(x)=\int_{0}^{\infty} \rho^{\prime}(x-y) u(y) d y=\int_{0}^{\infty} \rho^{\prime}(x-y)(u(y)-u(x)) d y
$$

$$
\left|v_{x}(x)\right|^{2} \leq \int_{0}^{\infty}\left|\rho^{\prime}(x-y)\right|(u(y)-u(x))^{2} d y \int_{0}^{\infty}\left|\rho^{\prime}(x-y)\right| d y .
$$

Using (5.7) and the fact that $\left|\rho^{\prime}(z)\right| \leq C(J)|J(z)|$ it follows that

$$
\int_{0}^{\infty}\left|v_{x}(x)\right|^{2} d x \leq\left\|\rho^{\prime}\right\|_{L^{1}(\mathbb{R})} \int_{0}^{\infty} \int_{0}^{\infty}\left|\rho^{\prime}(x-y)\right|(u(y)-u(x))^{2} d y d x \leq C(J) E_{I}^{J}(u, u) .
$$

On the other hand, since $\int_{-1}^{0} \rho=1$ and it is supported in $(-\infty, 0)$ we obtain

$$
\int_{0}^{\infty} \rho(x-y) d y=\int_{-\infty}^{x} \rho(z) d z=\int_{-\infty}^{0} \rho(z) d z
$$

and thus function $w=u-v$ can be written as

$$
w(x)=\int_{0}^{\infty}(u(x)-u(y)) \rho(x-y) d y, x>0,
$$

Using that $\left|\rho^{\prime}(z)\right| \leq C(J)|J(z)|$ and (5.7) it follows that $w$ satisfies

$$
\int_{0}^{\infty}|w(x)|^{2} d x \leq \int_{-\infty}^{0}|\rho(z)| d z \int_{0}^{\infty} \int_{0}^{\infty}|\rho(x-y)|(u(y)-u(x))^{2} d y d x \leq C(J) E_{I}^{J}(u, u) .
$$


This finishes the proof.

Lemma 5.2. Let $I$ be the half line $(0, \infty)$ or the real line $\mathbb{R}$. For any $p \in(1, \infty)$ and $u \in L^{p}(I)$ it holds

$$
\|u\|_{L^{p}(I)}^{p} \leq C(p, J)\left(\|u\|_{L^{1}(I)}^{\frac{2 p}{p+1}} E_{I}^{J}\left(|u|^{p / 2},|u|^{p / 2}\right)^{\frac{p-1}{p+1}}+E_{I}^{J}\left(|u|^{p / 2},|u|^{p / 2}\right)\right) .
$$

The case $p \geq 2$ follows from [11, Th. 1.1] so the our contribution here is to deal with the case $p \in(1,2)$. Extension to any dimension considering unbounded exterior domains can be done but it is out of the scope of this article.

Proof. Let us consider $p \in(1,2)$ since the other cases have been proved in [11. Let $u \in L^{p}(I)$. Then $|u|^{\frac{p}{2}} \in L^{2}(I)$. We consider the decomposition of $|u|^{\frac{p}{2}}=v+w$ as in Lemma 5.1] or [29, Th. 2.1] (when $I=\mathbb{R}$ ). In both cases $v=\rho *|u|^{\frac{p}{2}}:=\int_{I} \rho(x-y)|u|^{\frac{p}{2}}(y) d y$ and since $\frac{2}{p}>1$ we have

$$
\|v\|_{L^{\frac{2}{p}(I)}}=\left\|\rho *|u|^{\frac{p}{2}}\right\|_{L^{\frac{2}{p}(I)}} \leq\|\rho\|_{L^{1}(I)}\left\||u|^{\frac{p}{2}}\right\|_{L^{\frac{2}{p}(I)}} \leq C(J)\|u\|_{L^{1}(I)}^{\frac{p}{2}} .
$$

Also by Lemma 5.1

$$
\left\|v_{x}\right\|_{L^{2}(I)}^{2}+\|w\|_{L^{2}(I)}^{2} \leq C(J) E_{I}^{J}\left(|u|^{\frac{p}{2}},|u|^{\frac{p}{2}}\right) .
$$

Using the interpolation inequality and that $\|v\|_{L^{\infty}(I)}^{2} \leq 2\left\|v_{x}\right\|_{L^{2}(I)}\|v\|_{L^{2}(I)}$ we find

$$
\begin{aligned}
\|v\|_{L^{2}(I)} & \leq\|v\|_{L^{2 / p}(I)}^{\frac{1}{p}}\|v\|_{L^{\infty}(I)}^{\frac{p-1}{p}} \leq C(J)\|u\|_{L^{1}(I)}^{\frac{1}{2}}\left\|v_{x}\right\|_{L^{2}(I)}^{\frac{p-1}{2 p}}\|v\|_{L^{2}(I)}^{\frac{p-1}{2 p}} \\
& \leq C(J)\|u\|_{L^{1}(I)}^{\frac{1}{2}} E_{I}^{J}\left(|u|^{\frac{p}{2}},|u|^{\frac{p}{2}}\right)^{\frac{p-1}{4 p}}\|v\|_{L^{2}(I)}^{\frac{p-1}{2 p}} .
\end{aligned}
$$

It implies that

$$
\|v\|_{L^{2}(I)}^{2} \leq C(J)\|u\|_{L^{1}(I)}^{\frac{2 p}{p+1}} E_{I}^{J}\left(|u|^{\frac{p}{2}},|u|^{\frac{p}{2}}\right)^{\frac{p-1}{p+1}} .
$$

Finally we get

$$
\begin{aligned}
\|u\|_{L^{p}(I)}^{p} & =\left\||u|^{\frac{p}{2}}\right\|_{L^{2}(I)}^{2} \leq 2\left(\|v\|_{L^{2}(I)}^{2}+\|w\|_{L^{2}(I)}^{2}\right) \\
& \leq C(J)\left(\|u\|_{L^{1}(I)}^{\frac{2 p}{p+1}} E\left(|u|^{\frac{p}{2}},|u|^{\frac{p}{2}}\right)^{\frac{p-1}{p+1}}+E\left(|u|^{\frac{p}{2}},|u|^{\frac{p}{2}}\right)\right)
\end{aligned}
$$

which finishes the proof.

5.2.2. Decay of the solutions. In this section, our main result read as follows: we prove that the problem is well posed and a bound for the decay of solutions.

Theorem 5.2. For any $\mathbf{u}_{0} \in L^{1}(\Gamma) \cap L^{p}(\Gamma), 1 \leq p<\infty$, the solution $\mathbf{u}$ of system (5.1) satisfies

$$
\|\mathbf{u}(t)\|_{L^{p}(\Gamma)} \leq \frac{C\left(\left\|\mathbf{u}_{0}\right\|_{L^{1}(\Gamma)},\left\|\mathbf{u}_{0}\right\|_{L^{p}(\Gamma)}\right)}{(1+t)^{\frac{1}{2}\left(1-\frac{1}{p}\right)}}, \quad \forall t>0 .
$$

In addition, for any $\mathbf{u}_{0} \in L^{1}(\Gamma) \cap L^{2}(\Gamma)$

$$
\int_{\Gamma} \int_{\Gamma} J(d(x, y))(\mathbf{u}(t, x)-\mathbf{u}(t, y))^{2} d x d y \leq C\left(\left\|\mathbf{u}_{0}\right\|_{L^{1}(\Gamma)},\left\|\mathbf{u}_{0}\right\|_{L^{2}(\Gamma)}\right) t^{-\frac{3}{2}}
$$


Proof of Theorem 5.2. Observe that property (5.9) is a consequence of (5.2) and of the decay property (5.8). Indeed, by (5.2) the left hand side of (5.9) satisfies

$$
\mathcal{E}_{\Gamma}^{J}(\mathbf{u}(2 t), \mathbf{u}(2 t)) \leq \frac{\|\mathbf{u}(t)\|_{L^{2}(\Gamma)}^{2}}{t} \leq C\left(\left\|\mathbf{u}_{0}\right\|_{L^{1}(\Gamma)},\left\|\mathbf{u}_{0}\right\|_{L^{2}(\Gamma)}\right) t^{-\frac{3}{2}}
$$

We now prove the decay property (5.8). We use the results obtained in the previous section and the following energy estimate obtained by multiplying (5.1) with $|\mathbf{u}|^{p-2} \mathbf{u}$ and integrating in the space variable

$$
\begin{aligned}
\frac{1}{p} \frac{d}{d t} & \int_{\Gamma}|\mathbf{u}|^{p}(t, x) d x \\
& =-\frac{1}{2} \int_{\Gamma} \int_{\Gamma} J(x-y)(\mathbf{u}(t, x)-\mathbf{u}(t, y))\left(|\mathbf{u}(t, x)|^{p-2} \mathbf{u}(t, x)-|\mathbf{u}(t, y)|^{p-2} \mathbf{u}(t, y)\right) d x d y \\
& \leq-c(p) \int_{\Gamma} \int_{\Gamma} J(x-y)\left(|\mathbf{u}(t, x)|^{\frac{p}{2}}-|\mathbf{u}(t, y)|^{\frac{p}{2}}\right)^{2} d x d y=-c(p) \mathcal{E}_{\Gamma}^{J}\left(|\mathbf{u}(t)|^{\frac{p}{2}},|\mathbf{u}(t)|^{\frac{p}{2}}\right) .
\end{aligned}
$$

Let us now fix an edge $e$. For each such edge we choose a path $\Gamma_{e}$ that connects $e$ with $\Gamma_{\infty}$. It may happen to exist many such paths but we choose one of them. When an edge $e$ has infinite length we can chose $\Gamma_{e}$ to be exactly $e$. This path $\Gamma_{e}$ can be parametrized by the infinite interval $I_{e}=[0, \infty)$. We set $u_{e}$ to be the restriction of $\mathbf{u}$ to $\Gamma_{e}$. It is clear that

$$
\left\|u_{e}(t)\right\|_{L^{1}\left(\Gamma_{e}\right)} \leq\|\mathbf{u}(t)\|_{L^{1}(\Gamma)} \leq\left\|\mathbf{u}_{0}\right\|_{L^{1}(\Gamma)} .
$$

We apply Lemma 5.2 to each function $u_{e}(t)$ and obtain

$$
\begin{aligned}
\left\|u_{e}\right\|_{L^{p}\left(\Gamma_{e}\right)}^{p} & \leq C(p, J)\left(\left\|u_{e}\right\|_{L^{1}\left(\Gamma_{e}\right)}^{\frac{2 p}{p+1}} \mathcal{E}_{\Gamma_{e}}^{J}\left(\left|u_{e}\right|^{p / 2},\left|u_{e}\right|^{\frac{p}{2}}\right)^{\frac{p-1}{p+1}}+\mathcal{E}_{\Gamma_{e}}^{J}\left(\left|u_{e}\right|^{\frac{p}{2}},\left|u_{e}\right|^{\frac{p}{2}}\right)\right) \\
& \leq C(p, J)\left(\left\|\mathbf{u}_{0}\right\|_{L^{1}(\Gamma)}^{\frac{2 p}{p+1}} \mathcal{E}_{\Gamma}^{J}\left(|\mathbf{u}|^{\frac{p}{2}},|\mathbf{u}|^{\frac{p}{2}}\right)^{\frac{p-1}{p+1}}+\mathcal{E}_{\Gamma}^{J}\left(|\mathbf{u}|^{\frac{p}{2}},|\mathbf{u}|^{\frac{p}{2}}\right)\right) .
\end{aligned}
$$

Using that $u^{e}=\left(u_{e}\right)_{\left.\right|_{e}}=\mathbf{u}_{\mid e}$ and summing over all the edges (a finite number) of graph $\Gamma$ we get

$$
\begin{aligned}
\|\mathbf{u}(t)\|_{L^{p}(\Gamma)}^{p} & =\sum_{e \in E}\left\|u^{e}\right\|_{L^{p}(e)}^{p} \leq \sum_{e \in E}\left\|u_{e}\right\|_{L^{p}\left(\Gamma_{e}\right)}^{p} \\
& \leq|E| C(p, J)\left(\left\|\mathbf{u}_{0}\right\|_{L^{1}(\Gamma)}^{\frac{2 p}{p+1}} \mathcal{E}_{\Gamma}^{J}\left(|\mathbf{u}(t)|^{\frac{p}{2}},|\mathbf{u}(t)|^{\frac{p}{2}}\right)^{\frac{p-1}{p+1}}+\mathcal{E}_{\Gamma}^{J}\left(|\mathbf{u}|^{\frac{p}{2}},|\mathbf{u}|^{\frac{p}{2}}\right)\right) \\
& =f\left(\mathcal{E}_{\Gamma}^{J}\left(|\mathbf{u}|^{\frac{p}{2}},|\mathbf{u}|^{\frac{p}{2}}\right)\right),
\end{aligned}
$$

where

$$
f(s)=|E| C(p, J)\left(\left\|\mathbf{u}_{0}\right\|_{L^{1}(\Gamma)}^{\frac{2 p}{p+1}} s^{\frac{p-1}{p+1}}+s\right) .
$$

This shows that

$$
\frac{1}{p c(p)} \frac{d}{d t}\left(\|\mathbf{u}(t)\|_{L^{p}(\Gamma)}^{p}\right) \leq-f^{-1}\left(\|\mathbf{u}(t)\|_{L^{p}(\Gamma)}^{p}\right) .
$$

Using that $f(t) \simeq t^{\frac{p-1}{p+1}}$ as $t \simeq 0$ we obtain that $f^{-1}(t) \simeq t^{\frac{p+1}{p-1}}$ as $t \simeq 0$. The same arguments as in [29, Lemma 3.1] give us the desired decay estimate. 
5.2.3. Asymptotic behaviour. Now, let us rescale the solution as we did for the local case, let $\mathbf{u}_{\lambda}: \Gamma^{\lambda} \rightarrow \mathbb{R}, \lambda>0$ be given by

$$
\mathbf{u}_{\lambda}(t, x)=\lambda \mathbf{u}\left(\lambda^{2} t, \lambda x\right), \quad x \in \Gamma^{\lambda}, t>0 .
$$

It follows that $\mathbf{u}_{\lambda}$ satisfies

$$
\left\{\begin{array}{l}
\partial_{t} \mathbf{u}_{\lambda}(t, x)=\lambda^{3} \int_{\Gamma^{\lambda}} J(\lambda d(x, y))\left(\mathbf{u}_{\lambda}(t, y)-\mathbf{u}_{\lambda}(t, x)\right) d y, x \in \Gamma^{\lambda}, t>0 \\
\mathbf{u}_{\lambda}(x, 0)=\lambda \mathbf{u}_{0}(\lambda x), x \in \Gamma^{\lambda}
\end{array}\right.
$$

Using the estimates obtained in Theorem 5.2 for $\mathbf{u}$ we get that $\mathbf{u}_{\lambda}$ satisfies the following uniform estimates:

Lemma 5.3. Let $1 \leq p<\infty$. For any $\mathbf{u}_{0} \in L^{1}(\Gamma) \cap L^{p}(\Gamma)$ it holds that

$$
\left\|\mathbf{u}_{\lambda}(t)\right\|_{L^{p}\left(\Gamma^{\lambda}\right)} \leq C\left(p,\left\|\mathbf{u}_{0}\right\|_{L^{1}(\Gamma)},\left\|\mathbf{u}_{0}\right\|_{L^{p}(\Gamma)}\right) t^{-\frac{1}{2}\left(1-\frac{1}{p}\right)}, \forall t>0 .
$$

Moreover, for any $\mathbf{u}_{0} \in L^{1}(\Gamma) \cap L^{2}(\Gamma)$ the following bound

$$
\lambda^{3} \int_{\Gamma^{\lambda}} \int_{\Gamma^{\lambda}} J(\lambda d(x, y))\left(\mathbf{u}_{\lambda}(t, x)-\mathbf{u}_{\lambda}(t, y)\right)^{2} d x d y \leq C\left(p,\left\|\mathbf{u}_{0}\right\|_{L^{1}(\Gamma)},\left\|\mathbf{u}_{0}\right\|_{L^{2}(\Gamma)}\right) t^{-\frac{3}{2}}, \quad \forall t>0,
$$

holds uniformly in $\lambda>0$.

Before proving Theorem 3.3 we need some auxiliary results. For a function $\varphi \in H^{1}\left(\Gamma_{\infty}\right)$ such that $\varphi^{e}(0)=\varphi^{e^{\prime}}(0)$ for all $e, e^{\prime} \in \Gamma_{\infty}$, denote this common value by $\varphi(0)$. We extend it to function defined on the whole graph $\Gamma^{\lambda}, \tilde{\varphi}_{\lambda}: \Gamma^{\lambda} \rightarrow \mathbb{R}$, such that to be constant on the finite part of the graph, i.e.

$$
\tilde{\varphi}_{\lambda}= \begin{cases}\varphi, & \text { on } \Gamma_{\infty} \\ \varphi(0), & \text { on } \Gamma_{f}^{\lambda}\end{cases}
$$

Lemma 5.4. There exists a non-increasing function $H \in L^{\infty}([0, \infty))$ going to zero at infinity such that for any $\left.\varphi \in H^{1}\left(\Gamma_{\infty}\right)\right)$ such that $\varphi^{e}(0)=\varphi^{e^{\prime}}(0)$ for all $e, e^{\prime} \in \Gamma_{\infty}$ we have

$$
\lambda^{3} \int_{\Gamma_{\infty}} \int_{\Gamma_{f}^{\lambda}} J(\lambda d(x, y))(\tilde{\varphi}(x)-\tilde{\varphi}(y))^{2} d y d x \leq \int_{\Gamma_{\infty}} \varphi_{x}^{2}(x) H(\lambda|x|) d x, \quad \forall \lambda>0 .
$$

Proof. By the way we define the extension function $\tilde{\varphi}$ we have that it is constant on $\Gamma_{f}^{\lambda}$. Then

$$
\lambda^{3} \int_{\Gamma_{\infty}} \int_{\Gamma_{f}^{\lambda}} J(\lambda d(x, y))(\tilde{\varphi}(x)-\tilde{\varphi}(y))^{2} d y d x=\lambda^{3} \int_{\Gamma_{\infty}}(\varphi(x)-\varphi(0))^{2}\left(\int_{\Gamma_{f}^{\lambda}} J(\lambda d(x, y)) d y\right) d x .
$$

Take an edge $e$ of $\Gamma_{\infty}$ parametrized by $[0, \infty)$. For any $x \in e$ and $y \in \Gamma_{f}^{\lambda}$ we have $d(x, y) \geq|x|$. Since $J$ is a non-increasing function we obtain

$$
\int_{\Gamma_{f}^{\lambda}} J(\lambda d(x, y)) d y \leq\left|\Gamma_{f}^{\lambda}\right| J(\lambda|x|)=C\left(\Gamma_{f}\right) \lambda^{-1} J(\lambda|x|) .
$$

It is then sufficient to consider integrals of the type

$$
I=\lambda^{2} \int_{0}^{\infty}(\varphi(x)-\varphi(0))^{2} J(\lambda|x|) d x .
$$


Using that $(\varphi(x)-\varphi(0))^{2} \leq x \int_{0}^{x} \varphi_{x}^{2}(s) d s$ we obtain that

$$
I \leq \int_{0}^{\infty} \varphi_{x}^{2}(x) \int_{\lambda x}^{\infty} z J(z) d z .
$$

Denoting $H(s)=\int_{s}^{\infty} z J(z) d z$ and using that $J \in L^{1}\left(\mathbb{R}, 1+|x|^{2}\right)$ we obtain the desired result.

Lemma 5.5. For any $\varphi \in H^{1}\left(\Gamma_{\infty}\right)$ such that $\varphi^{e}(0)=\varphi^{e^{\prime}}(0)$ for all $e, e^{\prime} \in \Gamma_{\infty}$ the following hold:

i)

$$
\mathcal{E}_{\Gamma_{\infty}}^{J, \lambda}(\varphi, \varphi):=\lambda^{3} \int_{\Gamma_{\infty}} \int_{\Gamma_{\infty}} J(\lambda d(x, y))(\varphi(x)-\varphi(y))^{2} d y d x \leq C(J)\left\|\varphi_{x}\right\|_{L^{2}\left(\Gamma_{\infty}\right)}^{2},
$$

uniformly in $\lambda>0$.

ii)

$$
\mathcal{E}_{\Gamma^{\lambda}}^{J, \lambda}(\tilde{\varphi}, \tilde{\varphi}):=\lambda^{3} \int_{\Gamma^{\lambda}} \int_{\Gamma^{\lambda}} J(\lambda d(x, y))(\tilde{\varphi}(x)-\tilde{\varphi}(y))^{2} d y d x=\mathcal{E}_{\Gamma_{\infty}^{J, \lambda}}(\varphi, \varphi)+o(1), \quad \lambda \rightarrow \infty .
$$

Proof. Using the fact that the extension function $\tilde{\varphi}$ is constant on $\Gamma_{f}^{\lambda}$ we obtain

$$
\mathcal{E}_{\Gamma^{\lambda}}^{J, \lambda}(\tilde{\varphi}, \tilde{\varphi})=\mathcal{E}_{\Gamma_{\infty}}^{J, \lambda}(\varphi, \varphi)+2 \lambda^{3} \int_{\Gamma_{\infty}} \int_{\Gamma_{f}^{\lambda}} J(\lambda d(x, y))(\tilde{\varphi}(x)-\tilde{\varphi}(y))^{2} d y d x
$$

Using estimate (5.11) and the fact that $\varphi \in H^{1}\left(\Gamma_{\infty}\right)$ by Lebesgue convergence theorem we obtain that the last term is $o(1)$.

Let us now prove the first part. When $\Gamma_{\infty}$ consists only of one edge parametrized by $(0, \infty)$ we extend it to the whole line and use the previous results on the real line [26, Lemma 2.3].

Let us consider two edges $e$ and $e^{\prime}$ of $\Gamma_{\infty}$. It is sufficient to estimate each $I_{e, e^{\prime}}$ defined by

$$
I_{e, e^{\prime}}=\lambda^{3} \int_{e \cup e^{\prime}} \int_{e \cup e^{\prime}} J(\lambda d(x, y))(\varphi(x)-\varphi(y))^{2} d y d x .
$$

Assume that the two edges are parametrized by $(-\infty, 0]$ and $[0, \infty)$. Then $d(x, y) \geq|y-x|$ and using that $J$ is non-increasing we get (the continuity at $x=0$ guarantees that the function $\varphi$ belongs to $H^{1}(\mathbb{R})$ and we can apply then the result on the real line)

$$
I_{e, e^{\prime}} \leq \lambda^{3} \int_{\mathbb{R}} \int_{\mathbb{R}} J(\lambda|x-y|)(\varphi(x)-\varphi(y))^{2} d y d x \leq \int_{\mathbb{R}} J(z) z^{2} d z \int_{\mathbb{R}} \varphi_{x}^{2} d x .
$$

Summing this inequality over all the edges $e$ and $e^{\prime}$ of $\Gamma_{\infty}$ we obtain the desired estimate.

Now we are ready to proceed with the proof of the asymptotic behavior of the solutions. Notice that the obtained bound for the decay in $L^{p}$ obtained in Theorem 5.2 is the same that holds for the heat equation in $\Gamma$. Therefore, our next task is to show that we also have the same asymptotic profile.

Proof of Theorem 3.3. We consider the case $p \geq 2$ in order to avoid technical difficulties in order to obtain compactness. In particular $\mathbf{u}_{0} \in L^{1}(\Gamma) \cap L^{2}(\Gamma)$ and we can obtain the compactness of the rescaled family $\mathbf{u}_{\lambda}$ using the energy estimate (5.10) When $1<p<2$ we 
have to use the compactness arguments in [26] and to obtain new versions of the Lemmas in Section 6.

Step A. Existence of a limit profile $\mathbf{U} \in L_{l o c}^{2}\left((0, \infty), D\left(\mathcal{Q}_{\Gamma_{\infty}}\right)\right)$. Let us fix two times, $\tau$, $T$, such that $0<\tau<T<\infty$. Using the results in Lemma 5.3 and Lemma 6.2 we obtain that $\mathbf{U}=\left(U^{e}\right)_{e \in \Gamma_{\infty}} \in L^{2}\left((\tau, T), H^{1}\left(\Gamma_{\infty}\right)\right)$ and $\mathbf{u}_{\lambda} \rightarrow \mathbf{U}$ in $L^{2}\left((\tau, T), L_{l o c}^{2}\left(\Gamma_{\infty}\right)\right)$. Indeed using (5.10) for any edge $e$ of $\Gamma_{\infty}$ we obtain by Lemma 6.2 that $u^{e} \in L^{2}\left((\tau, T), H^{1}(e)\right), u_{\lambda}^{e}(t) \rightarrow U^{e}(t)$ in $L^{2}(e)$ and $u_{\lambda}^{e}(t) \rightarrow U^{e}(t)$ in $L^{2}\left((\tau, T), L_{l o c}^{2}(e)\right)$.

We prove that in fact the values of $\mathbf{U}$ at the endpoints of $e_{j}$ are equal, that is,

$$
U^{e}(t, j(I(e), e))=U^{e^{\prime}}\left(t, j\left(I\left(e^{\prime}\right), e^{\prime}\right)\right),
$$

for a.e. $t$ and then $\mathbf{U} \in L^{2}\left((\tau, T), D\left(\mathcal{Q}_{\Gamma_{\infty}}\right)\right)$. To do that let us consider two edges, $e$ and $e^{\prime}$, of $\Gamma_{\infty}$ and $\Upsilon^{\lambda}$ a path in $\Gamma^{\lambda}$ which contains the two edges. Since the graph $\Gamma_{f}$ is finite there exits $\alpha>0$ such that the length of the path $\Upsilon_{f}^{\lambda}=\Gamma^{\lambda} \cap \Upsilon^{\lambda}$ is $2 \alpha / \lambda$. Let us parametrize $e$ and $e^{\prime}$ with $(-\infty, 0)$ respectively $(0, \infty), \Upsilon_{f}^{\lambda}$ with $(-\alpha / \lambda, \alpha / \lambda)$. In the following we will not meke precise the time dependence of $\mathbf{u}_{\lambda}$ unless it is necesarely. We introduce the family $\left(f_{\lambda}\right)_{\lambda>0}$ defined by

$$
f_{\lambda}(x)= \begin{cases}u_{\lambda}^{e}(x+\alpha / \lambda), & x<-\alpha / \lambda, \\ \left.u_{\lambda}\right|_{\Upsilon_{f}^{\lambda}}(x), & |x|<\alpha / \lambda, \\ u_{\lambda}^{e^{\prime}}(x-\alpha / \lambda), & x>\alpha / \lambda .\end{cases}
$$

In view of the properties of $\mathbf{u}_{\lambda}(t)$ we obtain that $\left(f_{\lambda}\right)_{\lambda>0}$ is uniformly bounded in $L^{2}(\mathbb{R})$ and it safisfies

$$
\lambda^{3} \int_{\mathbb{R}} \int_{\mathbb{R}} J(\lambda(x-y))\left(f_{\lambda}(x)-f_{\lambda}(y)\right)^{2} d x d y \leq C(t) .
$$

It follows that there exists $f \in H^{1}(\mathbb{R})$ such that $f_{\lambda} \rightarrow f$ in $L_{\text {loc }}^{2}(\mathbb{R})$ and $f_{\lambda} \rightarrow f$ in $L^{2}(\mathbb{R})$. In particular $f \in C(\mathbb{R})$ and satisfies $|f(b)-f(a)| \lesssim|b-a|^{1 / 2}$ for all $a<0<b$. We claim that

$$
f(x)= \begin{cases}u^{e}(x), & x<0, \\ u^{e^{\prime}}(x), & x>0 .\end{cases}
$$

This implies that for any $a<0<b,\left|u^{e}(a)-u^{e^{\prime}}(b)\right| \lesssim|b-a|^{1 / 2}$ and then $u^{e}(0-)=u^{e^{\prime}}(0+)$ which proves the continuity of the limit profile $\mathbf{U}$. It remains to prove the above claim. Let us consider $a<0, \varphi$ a smooth function supported in $(-\infty, 0)$ and $\lambda>0$ such that $a<-\alpha / \lambda<0$. Then

$$
\int_{-\infty}^{a} f_{\lambda} \varphi=\int_{-\infty}^{a} u_{\lambda}^{e}\left(x+\frac{\alpha}{\lambda}\right) \varphi(x) d x=\int_{-\infty}^{0} u_{\lambda}^{e}(y) \varphi\left(y-\frac{\alpha}{\lambda}\right) \chi_{\left(-\infty, a+\frac{\alpha}{\lambda}\right)} \cdot d y
$$

Using that $f_{\lambda} \rightarrow f$ and $u_{\lambda}^{e} \rightarrow u^{e}$ in $L^{2}((-\infty, 0))$ we let $\lambda \rightarrow \infty$ to obtain that $f=u^{e}$ on $(-\infty, a)$. Since $a$ is arbitrary we obtain that $f \equiv u_{e}$ on $(-\infty, 0)$. The same argument shows that $f \equiv u_{e^{\prime}}$ on $(0, \infty)$ and the claim is proved.

Step B. Equation satisfied by the limit profile. Let us fix $T>0$. Let us now consider a function $\varphi \in C\left([0, \infty), H^{1}\left(\Gamma_{\infty}\right)\right)$ with $\varphi_{t} \in C\left([0, \infty), L^{2}\left(\Gamma_{\infty}\right)\right)$ compactly supported in time in the interval $[0, T]$ and such that $\varphi^{e}(t, 0)=\varphi^{e^{\prime}}(t, 0)$ for all $e, e^{\prime} \in \Gamma_{\infty}$, i.e. $\varphi \in$ $C\left([0, \infty), D\left(\mathcal{Q}_{\Gamma_{\infty}}\right)\right)$. Denote this common value by $\varphi(t, 0)$. We extend it to function defined 
on the whole graph $\Gamma^{\lambda}, \tilde{\varphi}_{\lambda}:[0, \infty) \times \Gamma^{\lambda}$, function that is constant on the finite part of the graph, i.e.

$$
\tilde{\varphi}_{\lambda}= \begin{cases}\varphi, & \text { on } \Gamma_{\infty}, \\ \varphi(t, 0), & \text { on } \Gamma_{f}^{\lambda} .\end{cases}
$$

Multiplying the equation satisfied by $\mathbf{u}_{\lambda}$ with $\varphi$ and integrating in time and space we obtain

$$
\begin{aligned}
0= & \int_{0}^{T} \int_{\Gamma^{\lambda}} \mathbf{u}_{\lambda} \varphi_{t}(t, x) d x d t+\int_{\Gamma^{\lambda}} \mathbf{u}_{\lambda}(0, x) \varphi_{\lambda}(0, x) d x d t \\
& +\lambda^{3} \int_{0}^{T} \int_{\Gamma^{\lambda}} \int_{\Gamma^{\lambda}} J(\lambda d(x, y))\left(\mathbf{u}_{\lambda}(t, y)-\mathbf{u}_{\lambda}(t, x)\right) \varphi(t, x) d y d x d t \\
= & I_{1}^{\lambda}+I_{2}^{\lambda}-\frac{1}{2} \int_{0}^{T} \mathcal{E}_{\Gamma^{\lambda}}^{J, \lambda}\left(\mathbf{u}_{\lambda}(t), \varphi(t)\right) d t:=I_{1}^{\lambda}+I_{2}^{\lambda}+I_{3}^{\lambda}
\end{aligned}
$$

where

$$
\mathcal{E}_{\Gamma^{\lambda}}^{J, \lambda}\left(\mathbf{u}_{\lambda}(t), \varphi\right)=\lambda^{3} \int_{\Gamma^{\lambda}} \int_{\Gamma^{\lambda}} J(\lambda d(x, y))\left(\mathbf{u}_{\lambda}(t, y)-\mathbf{u}_{\lambda}(t, x)\right)(\varphi(t, y)-\varphi(t, x)) d x d y .
$$

We claim that the following hold where $\mathbf{U} \in L_{\text {lov }}^{2}\left((0, \infty), D\left(\mathcal{Q}_{\Gamma_{\infty}}\right)\right)$ is the a limit of $\mathbf{u}_{\lambda}$ above

$$
\begin{gathered}
I_{1}^{\lambda} \rightarrow \int_{0}^{T} \int_{\Gamma_{\infty}} \mathbf{U} \varphi_{t}, \\
I_{2}^{\lambda} \rightarrow \varphi(0,0) M, \\
I_{3}^{\lambda} \rightarrow-\frac{1}{2} \int_{\mathbb{R}} J(z) z^{2} d z \int_{0}^{T} \int_{\Gamma_{\infty}} \mathbf{U}_{x} \varphi_{x}=-\int_{0}^{T} \int_{\Gamma_{\infty}} \mathbf{U}_{x} \varphi_{x} .
\end{gathered}
$$

These convergences show that the limit function $\mathbf{U} \in L_{l o c}^{2}\left((0, \infty), D\left(\mathcal{Q}_{\Gamma_{\infty}}\right)\right)$ is a solution to

$$
0=\int_{0}^{\infty} \int_{\Gamma_{\infty}} \mathbf{U} \varphi_{t}+\varphi(0,0) M-\int_{0}^{\infty} \int_{\Gamma_{\infty}} \mathbf{U}_{x} \varphi_{x}
$$

Since $\mathbf{U}(t) \in D\left(\mathcal{Q}_{\Gamma_{\infty}}\right)$ for a.e. $t>0$ we have for any $\varphi \in D\left(\Delta_{\Gamma_{\infty}}\right)$ that

$$
\left(\mathbf{U}_{x}, \varphi_{x}\right)_{L^{2}\left(\Gamma_{\infty}\right)}=\left(\mathbf{U}, \varphi_{x x}\right)_{L^{2}\left(\Gamma_{\infty}\right)} .
$$

Thus for $\varphi \in C\left([0, \infty), D\left(\Delta_{\Gamma_{\infty}}\right)\right) \cap C^{1}\left([0, \infty), L^{2}\left(\Gamma_{\infty}\right)\right)$ we obtain that the limit point $\mathbf{U}$ satisfies

$$
0=\int_{0}^{\infty} \int_{\Gamma_{\infty}} \mathbf{U}(t, x)\left(\varphi_{t}(t, x)+\varphi_{x x}(t, x)\right) d x d t+\varphi(0,0) M,
$$

hence it is a solution to the heat equation in $\Gamma$ with initial condition $M \delta_{x=0}$ and therefore the asymptotic profile claimed in Theorem 3.3 follows from our results for the local case.

Therefore, we have to show (5.12), (5.13) and (5.14). As in the local case we have that $\mathbf{U}$ is uniformly bounded in $L^{2}\left((0, T), L^{2}\left(\Gamma_{\infty}\right)\right)$ so

$$
\int_{0}^{\infty} \int_{\Gamma_{\infty}} \mathbf{u}_{\lambda} \widetilde{\varphi}_{t} \rightarrow \int_{0}^{\infty} \int_{\Gamma_{\infty}} \mathbf{U} \varphi_{t}
$$


Moreover, since $\varphi_{t}(t, 0)$ has compact support in time

$$
\int_{0}^{\infty} \int_{\Gamma_{f}^{\lambda}}\left|\mathbf{u}_{\lambda} \widetilde{\varphi}_{t}\right| d x d t \leq \int_{0}^{\infty}\left\|u_{\lambda}(t)\right\|_{L^{2}\left(\Gamma_{f}^{\lambda}\right)}\left|\varphi_{t}(t, 0)\right|\left|\Gamma_{f}^{\lambda}\right|^{1 / 2} \lesssim \lambda^{-\frac{1}{2}} \int_{0}^{\infty} t^{-1 / 4}\left|\varphi_{t}(t, 0)\right| d t \rightarrow 0 .
$$

This shows (5.12).

For the second estimate we use the mass conservation an the fact that $\tilde{\varphi}_{\lambda}$ is constant on $\Gamma_{f}^{\lambda}$ :

$$
\begin{aligned}
I_{\lambda}^{2}-M \varphi(0,0) & =\int_{\Gamma^{\lambda}} u^{\lambda}(0, x)\left(\tilde{\varphi}_{\lambda}(0, x)-\varphi(0,0)\right) d x=\int_{\Gamma_{\infty}} u^{\lambda}(0, x)(\varphi(0, x)-\varphi(0,0)) d x \\
& =\int_{\Gamma_{\infty}} u(0, x)(\varphi(0, x / \lambda)-\varphi(0,0)) d x \rightarrow 0 .
\end{aligned}
$$

Let us analyze the last term $I_{3}^{\lambda}$. We will prove the desired limit in few steps.

Step 1. We prove that

$$
\left.\int_{0}^{T} \mathcal{E}_{\Gamma^{\lambda}}^{J, \lambda}\left(\mathbf{u}_{\lambda}(t), \tilde{\varphi}(t)\right) d t=\int_{0}^{T} \mathcal{E}_{\Gamma_{\infty}^{J, \lambda}}^{J \mathbf{u}_{\lambda}}(t), \varphi(t)\right) d t+o(1) .
$$

Indeed, since $\tilde{\varphi}$ is constant in $\Gamma_{f}^{\lambda}$ we have

$$
\begin{aligned}
& \left|\mathcal{E}_{\Gamma^{\lambda}}^{J, \lambda}\left(\mathbf{u}_{\lambda}(t), \tilde{\varphi}(t)\right)-\mathcal{E}_{\Gamma_{\infty}}^{J, \lambda}\left(\mathbf{u}_{\lambda}(t), \varphi(t)\right)\right| \\
& \leq 2 \lambda^{3} \int_{\Gamma_{\infty}} \int_{\Gamma_{f}^{\lambda}} J(\lambda d(x, y))\left|\mathbf{u}_{\lambda}(t, x)-\mathbf{u}_{\lambda}(t, y)\right||\tilde{\varphi}(t, x)-\tilde{\varphi}(t, y)| d y d x \\
& \leq 2\left(\mathcal{E}_{\Gamma^{\lambda}}^{J, \lambda}\left(\mathbf{u}_{\lambda}(t),\left(\mathbf{u}_{\lambda}(t)\right)\right)^{1 / 2}\left(\lambda^{3} \int_{\Gamma_{\infty}} \int_{\Gamma_{f}^{\lambda}} J(\lambda d(x, y))(\tilde{\varphi}(x)-\tilde{\varphi}(y))^{2} d y d x\right)^{\frac{1}{2}}\right. \\
& \lesssim t^{-\frac{3}{4}}\left(\int_{\Gamma_{\infty}} \varphi_{x}^{2}(t, x) H(\lambda x) d x\right)^{\frac{1}{2}} .
\end{aligned}
$$

Integrating in time the above inequality we obtain that

$$
\int_{0}^{T}\left|\mathcal{E}_{\Gamma^{\lambda}}^{J, \lambda}\left(\mathbf{u}_{\lambda}(t), \tilde{\varphi}(t)\right)-\mathcal{E}_{\Gamma_{\infty}^{J}}^{J, \lambda}\left(\mathbf{u}_{\lambda}(t), \varphi(t)\right)\right| d t \lesssim T^{\frac{1}{4}} \sup _{t \in[0, T]}\left(\int_{\Gamma_{\infty}} \varphi_{x}^{2}(t, x) H(\lambda x) d x\right)^{\frac{1}{2}} .
$$

Using that $\varphi \in C\left([0, \infty), H^{1}\left(\Gamma_{\infty}\right)\right)$ we can apply the dominated convergen theorem to obtain that the last goes to zero as $\lambda \rightarrow \infty$.

Step 2. For any $0<\tau<T$ the integrals over $(0, \tau)$ are small, uniformly in $\lambda$ : it holds that

$$
\int_{0}^{\tau}\left|\mathcal{E}_{\Gamma_{\infty}}^{J, \lambda}\left(\mathbf{u}_{\lambda}(t), \varphi(t)\right)\right| d t \lesssim \tau^{\frac{1}{4}}\|\varphi\|_{L^{\infty}\left([0, T], H^{1}\left(\Gamma_{\infty}\right)\right)}
$$

and

$$
\left|\int_{0}^{\tau} \int_{\Gamma_{\infty}} \mathbf{U}_{x}(t, x) \varphi_{x}(t, x) d x d t\right| \leq \tau^{\frac{1}{4}}\|\varphi\|_{L^{\infty}\left([0, T], H^{1}\left(\Gamma_{\infty}\right)\right)}
$$

To check the first one notice that, in view of (5.10), we have

$$
\left|\mathcal{E}_{\Gamma_{\infty}}^{J, \lambda}\left(\mathbf{u}_{\lambda}(t), \varphi(t)\right)\right| \leq \mathcal{E}_{\Gamma_{\infty}}^{J, \lambda}\left(\mathbf{u}_{\lambda}(t), \mathbf{u}_{\lambda}(t)\right)^{\frac{1}{2}} \mathcal{E}_{\Gamma_{\infty}}^{J, \lambda}(\varphi(t), \varphi(t))^{\frac{1}{2}} \lesssim t^{-\frac{3}{4}}\left\|\varphi_{x}(t)\right\|_{L^{2}\left(\Gamma_{\infty}\right)} .
$$


Integrating in $[0, \tau]$ we obtain the desired estimate.

For the second limit remark that for each $t>0$, we have

$$
\mathcal{E}_{\Gamma_{\infty}}^{\lambda}\left(\mathbf{u}_{\lambda}(t), \varphi(t)\right) \rightarrow \int_{\Gamma_{\infty}} \mathbf{U}_{x}(t, x) \varphi_{x}(t, x) d x
$$

Also $\left|\mathcal{E}_{\Gamma_{\infty}}^{\lambda}\left(\mathbf{u}_{\lambda}(t), \varphi(t)\right)\right| \leq t^{-\frac{3}{4}}\|\varphi\|_{L^{\infty}\left([0, T], H^{1}\left(\Gamma_{\infty}\right)\right)} \in L^{1}((0, T))$. The dominated convergence theorem applied on the time interval $(0, \tau)$ gives us the desired result.

Step 3. Let us choose $0<\tau<T$. On the interval $[\tau, T]$ we apply the third part of Lemma 6.2 to $D=(0, \infty)$ to obtain that

$$
\int_{\tau}^{T} \mathcal{E}_{\Gamma_{\infty}}^{\lambda}\left(\mathbf{u}_{\lambda}(t), \varphi(t)\right) d t \rightarrow \int_{\mathbb{R}} J(z) z^{2} d z \int_{\tau}^{T} \int_{\Gamma_{\infty}} U_{x}(t, x) \varphi_{x}(t, x) d x d t .
$$

Thus in view of Step II we obtain (5.14).

Step C. Tail control and conclusion. Using the arguments for nonlocal problems in [26, Lemma 2.7] together with the ones in Lemma 4.1 to control the tail (4.11) we obtain similar results for the solutions $\mathbf{u}_{\lambda}$ of the nonlocal problem. It means that the local convergence obtained at Step A is not only local but it holds in $L^{1}\left(\Gamma_{\infty}\right)$ : for some $t_{0}>0$ it holds

$$
\mathbf{u}_{\lambda}\left(t_{0}\right) \rightarrow \mathbf{U}_{M}\left(t_{0}\right) \text { in } L^{1}\left(\Gamma_{\infty}\right) .
$$

Then (3.11) holds for $q=1$. The other cases follows by using the strong convergence in $L^{1}\left(\Gamma_{\infty}\right)$ together with the decay of the solutions in $L^{p}(\Gamma)$. Indeed, choosing $\alpha$ such that

$$
\frac{1}{q}=\frac{\alpha}{p}+\frac{1-\alpha}{1}
$$

we obtain

$$
\begin{aligned}
\left\|\mathbf{u}(t)-\mathbf{U}_{M}(t)\right\|_{L^{q}\left(\Gamma_{\infty}\right)} & \leq\left\|\mathbf{u}(t)-\mathbf{U}_{M}(t)\right\|_{L^{1}\left(\Gamma_{\infty}\right)}^{1-\alpha}\left\|\mathbf{u}(t)-\mathbf{U}_{M}(t)\right\|_{L^{p}\left(\Gamma_{\infty}\right)}^{\alpha} \\
& \leq o(1) t^{-\frac{1}{2}\left(1-\frac{1}{q}\right)}
\end{aligned}
$$

On the compact part of the graph for any $1 \leq q<p$ we trivially have

$$
\begin{aligned}
\left\|\mathbf{u}(t)-\mathbf{U}_{M}(t)\right\|_{L^{q}\left(\Gamma_{f}\right)} & \leq C\left(p, q, \Gamma_{f}\right)\left\|\mathbf{u}(t)-\mathbf{U}_{M}(t)\right\|_{L^{p}\left(\Gamma_{f}\right)} \\
& \leq C\left(p, q, \Gamma_{f}\right)\left(\|\mathbf{u}(t)\|_{L^{p}(\Gamma)}+\left\|\mathbf{U}_{M}(t)\right\|_{L^{p}\left(\Gamma_{f}\right)}\right) \\
& \leq C\left(p, q, \Gamma_{f}\right)\left(t^{-\frac{1}{2}\left(1-\frac{1}{p}\right)}+t^{-\frac{1}{2}}\right)=o\left(t^{-\frac{1}{2}\left(1-\frac{1}{q}\right)}\right), t \rightarrow \infty .
\end{aligned}
$$

The proof is now complete.

\section{Appendix}

In this Appendix we collect some compactness results that were used when studying the relaxation limit and the asymptotic behaviour for the nonlocal problem. We will use these results in 1 -dimension (take $d=1$ below) but we state them in any dimension since the results hold with greater generality. For the proof we use ideas from [1]. More general assumptions on the function $\rho$ can be found in [40]. 
Lemma 6.1. Let $D \subset \mathbb{R}^{d}$ be an open set that has the extension property in $H^{1}$, for example, $D$ is a bounded $C^{1}$-domain or $\mathbb{R}_{+}^{d}$. Let $\rho: \mathbb{R}^{d} \rightarrow \mathbb{R}$ be a nonnegative $L^{1}$ radial function having a second momentum in $L^{1}\left(\mathbb{R}^{d}\right), \rho>0$ in a neighbourhood of $x=0$, and take

$$
\rho_{n}(x)=n^{d} \rho(n x) .
$$

Let $f_{n}$ be a sequence in $L^{2}(D)$ such that

$$
n^{2} \int_{D} \int_{D} \rho_{n}(x-y)\left(f_{n}(x)-f_{n}(y)\right)^{2} d x d y \leq M .
$$

1. If $f_{n} \rightarrow f$ in $L^{2}(D)$ then $f \in H^{1}(D)$ and

$$
F_{n}(x, z)=(\rho(z))^{\frac{1}{2}} \chi_{D}\left(x+\frac{z}{n}\right) \frac{f_{n}\left(x+\frac{z}{n}\right)-f_{n}(x)}{\frac{1}{n}} \rightarrow(\rho(z))^{\frac{1}{2}} z \cdot \nabla f(x)
$$

weakly in $L_{x}^{2}(D) \times L_{z}^{2}\left(\mathbb{R}^{d}\right)$.

2. For any $f \in H^{1}(D)$

$$
\int_{\mathbb{R}^{d}} \rho(z)|z|^{2} d z \int_{D}|\nabla f(x)|^{2} d x \leq \liminf _{n \rightarrow \infty} n^{2} \int_{D} \int_{D} \rho_{n}(x-y)(f(x)-f(y))^{2} d x d y
$$

3. If $\varphi \in H^{1}\left(\mathbb{R}^{d}\right)$ then

$$
\begin{aligned}
n^{2} \int_{D} \int_{D} \rho_{n}(x-y)\left(f_{n}(x)-f_{n}(y)\right) & (\varphi(x)-\varphi(y)) d x d y \\
& \rightarrow \int_{\mathbb{R}^{d}} \rho(z)|z|^{2} d z \int_{D} \nabla f(x) \nabla \varphi(x) d x
\end{aligned}
$$

Notice that by 1 . we have $f \in H^{1}(D)$.

4. If $D$ is a smooth bounded domain of $\mathbb{R}^{d}$ and $\rho(x) \geq \rho(y)$ if $|x| \leq|y|$ then $\left\{f_{n}\right\}_{n}$ is relatively compact in $L^{2}(D)$.

Proof. The first and fourth part are in [1, Th. 6.11, p. 128]. The second part is exactly equation (36) in [8].

For the third part we claim that the following strong convergence holds in $L_{x}^{2}(D) \times L_{z}^{2}\left(\mathbb{R}^{d}\right)$ :

$$
\eta_{n}=(\rho(z))^{\frac{1}{2}} \chi_{D}\left(x+\frac{z}{n}\right) \frac{\varphi\left(x+\frac{z}{n}\right)-\varphi(x)}{\frac{1}{n}} \rightarrow(\rho(z))^{\frac{1}{2}} z \cdot \nabla \varphi(x) .
$$

Then

$$
\left(F_{n}, \eta_{n}\right)_{L_{x}^{2}(D) \times L_{z}^{2}\left(\mathbb{R}^{d}\right)} \rightarrow \int_{D_{x}} \int_{\mathbb{R}^{d}} \rho(z)(z \cdot \nabla f(x))(z \cdot \nabla \varphi(x))=\int_{\mathbb{R}^{d}} \rho(z)|z|^{2} d z \int_{D} \nabla f \cdot \nabla \varphi .
$$

Observe that after the change of variables $x+z / n=y$ we get

$$
\begin{aligned}
\left(F_{n}, \eta_{n}\right)_{L_{x}^{2}(D) \times L_{z}^{2}\left(\mathbb{R}^{d}\right)} & =n^{2} \int_{D} \int_{\mathbb{R}^{d}} \rho(z) \chi_{D}\left(x+\frac{z}{n}\right)\left(f_{n}\left(x+\frac{z}{n}\right)-f_{n}(x)\right)\left(\varphi\left(x+\frac{z}{n}\right)-\varphi(x)\right) d z d x \\
& =n^{d+2} \int_{D} \int_{\mathbb{R}^{d}} \rho(n(y-x)) \chi_{D}(y)\left(f_{n}(y)-f_{n}(x)\right)(\varphi(y)-\varphi(x)) d y d x \\
& =n^{2} \int_{D} \int_{D} \rho_{n}(y-x)\left(f_{n}(y)-f_{n}(x)\right)(\varphi(y)-\varphi(x)) d y d x
\end{aligned}
$$


which proves (6.15). It remains to prove the claim (6.16). To this end notice that

$$
\begin{aligned}
\int_{D} \int_{\mathbb{R}^{d}} \rho(z) & \left|\frac{\varphi(x+\varepsilon z)-\varphi(x)}{\varepsilon} \chi(x+\varepsilon z)-z \nabla \varphi(x)\right|^{2} d z d x \\
\lesssim & \int_{D} \int_{\mathbb{R}^{d}} \rho(z)\left|\frac{\varphi(x+\varepsilon z)-\varphi(x)}{\varepsilon}-z \nabla \varphi(x)\right|^{2} \chi(x+\varepsilon z) d z d x \\
& +\int_{D} \int_{\mathbb{R}^{d}} \rho(z)|z \nabla \varphi(x)|^{2}|\chi(x+\varepsilon z)-1|^{2} d z d x .
\end{aligned}
$$

The last term goes to zero thanks to the fact that $|z|^{2} \rho(z)|\nabla \varphi(x)|^{2} \in L^{1}\left(D \times \mathbb{R}^{d}\right)$ and the dominated convergence theorem. For the first term we use the Fourier transform and again the dominated convergence theorem to obtain that

$$
\begin{aligned}
\int_{D} \int_{\mathbb{R}^{d}} \rho(z) & \left|\frac{\varphi(x+\varepsilon z)-\varphi(x)}{\varepsilon}-z \nabla \varphi(x)\right|^{2} d z d x \\
\leq & \int_{\mathbb{R}^{d}} \int_{\mathbb{R}^{d}} \rho(z) \int_{\mathbb{R}^{d}}\left|\frac{e^{2 \pi i \varepsilon \xi z}-1}{\varepsilon}-2 \pi i \xi z\right|^{2}|\hat{\varphi}(\xi)|^{2} d \xi \rightarrow 0, \quad \varepsilon \rightarrow 0 .
\end{aligned}
$$

The proof is now finished.

In our analysis we need a version of the last lemma that will involve also integrals in time and implies convergences in $L^{2}((0, T) \times D)$. This is inspired in [26]. The proof follows the same ideas of the previous lemma and hence it is omitted.

Lemma 6.2. Let $D \subset \mathbb{R}^{d}$ be an open set with the extension property. Let $\rho: \mathbb{R}^{d} \rightarrow \mathbb{R}$ be a nonnegative $L^{1}$ radial function having a second momentum in $L^{1}\left(\mathbb{R}^{d}\right), \rho>0$ in neighbourhood of $x=0$, and $\rho_{n}(x)=n^{d} \rho(n x)$. Let $f_{n}$ be a sequence in $L^{2}((0, T) \times D)$ such that

$$
n^{2} \int_{0}^{T} \int_{D} \int_{D} \rho_{n}(x-y)\left(f_{n}(x)-f_{n}(y)\right)^{2} d x d y \leq M .
$$

1. If $f_{n} \rightarrow f$ in $L^{2}((0, T) \times D)$ then $f \in L^{2}\left((0, T), H^{1}(D)\right)$ and

$$
F_{n}(x, z)=(\rho(z))^{\frac{1}{2}} \chi_{D}\left(x+\frac{z}{n}\right) \frac{f_{n}\left(x+\frac{z}{n}\right)-f_{n}(x)}{\frac{1}{n}} \rightarrow(\rho(z))^{\frac{1}{2}} z \cdot \nabla f(x)
$$

weakly in $L^{2}\left((0, T), L_{x}^{2}(D) \times L_{z}^{2}\left(\mathbb{R}^{d}\right)\right)$.

2. For any $f \in L^{2}\left((0, T), H^{1}(D)\right)$

$$
\int_{\mathbb{R}^{d}} \rho(z)|z|^{2} d z \int_{0}^{T} \int_{D} \nabla f(x) \nabla \varphi(x) d x \leq \liminf _{n \rightarrow \infty} n^{2} \int_{0}^{T} \int_{D} \int_{D} \rho_{n}(x-y)(f(x)-f(y))^{2} d x d y
$$

3. If $\varphi \in L^{2}\left((0, T), H^{1}\left(\mathbb{R}^{d}\right)\right)$ then

$$
\begin{aligned}
n^{2} \int_{0}^{T} \int_{D} \int_{D} \rho_{n}(x-y)\left(f_{n}(x)-f_{n}(y)\right) & (\varphi(x)-\varphi(y)) d x d y \\
& \rightarrow \int_{\mathbb{R}^{d}} \rho(z)|z|^{2} d z \int_{0}^{T} \int_{D} \nabla f(x) \nabla \varphi(x) d x
\end{aligned}
$$

Notice that by 1 . we have $f \in L^{2}\left((0, T), H^{1}(D)\right)$. 
4. If $D$ is a smooth bounded domain of $\mathbb{R}^{d}, \rho(x) \geq \rho(y)$ if $|x| \leq|y|$ and $\left\|\partial_{t} f_{n}\right\|_{L^{2}\left((0, T), H^{-1}(D)\right)}$ is uniformly bounded then $\left\{f_{n}\right\}_{n}$ is relatively compact in $L^{2}(D)$.

Finally, we include a lemma in the one dimensional case.

Lemma 6.3. Let $\rho$ a nonincreasing $L^{1}$ radially symmetric function with $|r|^{2} \rho(r) \in L^{1}(\mathbb{R})$. Let $-\infty \leq a<0<b \leq+\infty$. Then, for any $\varphi \in H^{1}(a, b)$, it holds that

$$
\varepsilon^{-2} \lim _{\varepsilon \rightarrow 0} \int_{a}^{0} \int_{0}^{b} \rho_{\varepsilon}(x-y)(\varphi(x)-\varphi(y))^{2} d x d y=0 .
$$

Proof. We use (6.17) (see also [8, Th. 2, Remark 5]) to obtain that

$$
\left\|r^{2} \rho(r)\right\|_{L^{1}(\mathbb{R})} \int_{I} f_{x}^{2} \leq \liminf _{\varepsilon \rightarrow 0} \varepsilon^{-2} \int_{I} \int_{I} \rho_{\varepsilon}(x-y)(f(x)-f(y))^{2} d x d y,
$$

holds for for any interval $I$ of $\mathbb{R}$ and $f \in H^{1}(I)$. Moreover, applying (6.18) to $f_{n}=\varphi=f$ we have equality when $I=\mathbb{R}^{d}$.

Since we are in dimension one, any function $\varphi \in H^{1}(a, b)$ can be extended to a function $\widetilde{\varphi} \in H^{1}(\mathbb{R})$. Then

$$
\begin{aligned}
2 \varepsilon^{-2} \int_{a}^{0} \int_{0}^{b} \rho_{\varepsilon}(x-y)(\varphi(x)-\varphi(y))^{2} d x d y \leq 2 \varepsilon^{-2} \int_{-\infty}^{0} \int_{0}^{\infty} \rho_{\varepsilon}(x-y)(\widetilde{\varphi}(x)-\widetilde{\varphi}(y))^{2} d x d y \\
=\varepsilon^{-2} \int_{\mathbb{R}} \int_{\mathbb{R}} \rho_{\varepsilon}(x-y)(\widetilde{\varphi}(x)-\widetilde{\varphi}(y))^{2} d x d y \\
\quad-\varepsilon^{-2} \int_{-\infty}^{0} \int_{-\infty}^{0} \rho_{\varepsilon}(x-y)(\widetilde{\varphi}(x)-\widetilde{\varphi}(y))^{2} d x d y \\
\quad-\varepsilon^{-2} \int_{0}^{\infty} \int_{0}^{\infty} \rho_{\varepsilon}(x-y)(\widetilde{\varphi}(x)-\widetilde{\varphi}(y))^{2} d x d y
\end{aligned}
$$

Using (6.19) with $I=(-\infty, 0)$ and $I=(-\infty, 0)$ as well as the fact that it becomes equality when $I=\mathbb{R}$ we get that

$$
\begin{aligned}
\limsup _{\varepsilon \rightarrow 0} 2 \varepsilon^{-2} & \int_{a}^{0} \int_{0}^{b} \rho_{\varepsilon}(x-y)(\varphi(x)-\varphi(y))^{2} d x d y \\
& \leq\left\|r^{2} \rho(r)\right\|_{L^{1}(\mathbb{R})}\left[\int_{\mathbb{R}} \varphi_{x}^{2}-\int_{-\infty}^{0} \varphi_{x}^{2}-\int_{0}^{\infty} \varphi_{x}^{2}\right]=0
\end{aligned}
$$

which finishes the proof.

\section{Acknowledgements.}

Part of this work was done during a visit of JDR to the Institute of Mathematics "Simion Stoilow" at Bucharest, he is grateful for the friendly and stimulating working atmosphere found there.

L. I. was partially supported by a grant of Ministry of Research and Innovation, CNCSUEFISCDI, project PN-III-P1-1.1-TE-2016- 2233, within PNCDI III. J.D.R. partially supported by CONICET grant PIP GI No 11220150100036CO (Argentina), PICT-2018-03183 (Argentina) and UBACyT grant 20020160100155BA (Argentina). 


\section{REFERENCES}

[1] F. Andreu-Vaillo, J. M. Mazon, J. D. Rossi, and J. J. Toledo-Melero, Nonlocal diffusion problems. Mathematical Surveys and Monographs, 165. American Mathematical Society, Providence, RI; Real Sociedad Matemática Española, Madrid, 2010. xvi+256 pp.

[2] W. Arendt, Semigroups and evolution equations: functional calculus, regularity and kernel estimates, Evolutionary equations., Vol. I, Handb. Differ. Equ., 1-85, North-Holland, Amsterdam, 2004.

[3] G. Berkolaiko and P. Kuchment. Introduction to quantum graphs, volume 186 of Mathematical Surveys and Monographs. American Mathematical Society, Providence, RI, 2013.

[4] G. Berkolaiko, Y. Latushkin, and S. Sukhtaiev. Limits of quantum graph operators with shrinking edges, 2019. Adv. Math. 352, (2019), 632-669.

[5] J. Bourgain, H. Brezis and P. Mironescu. Another look at Sobolev spaces. In Optimal control and partial differential equations, pages 439-455. IOS, Amsterdam, 2001.

[6] H. Brézis and A. Friedman. Nonlinear parabolic equations involving measures as initial conditions. J. Math. Pures Appl. (9), 62(1), (1983), 73-97.

[7] M. Bodnar and J.J.L. Velazquez. An integro-differential equation arising as a limit of individual cell-based models. J. Differential Equations, 222, (2006), 341-380.

[8] H. Brezis. How to recognize constant functions. A connection with Sobolev spaces. Uspekhi Mat. Nauk, $57(4(346)),(2002), 59-74$.

[9] C. Cacciapuoti. Scale invariant e?ective hamiltonians for a graph with a small compact core. Symmetry, 11:359, 2019.

[10] C. Carrillo and P. Fife. Spatial effects in discrete generation population models. J. Math. Biol., 50(2), (2005), 161-188.

[11] J. A. Canizo and A. Molino, Alexis. Improved energy methods for nonlocal diffusion problems. Discrete Contin. Dyn. Syst. 38 (2018), no. 3, 1405-1425.

[12] C. Cazacu, L. I. Ignat and A. Pazoto. On the asymptotic behavior of a subcritical convection-diffusion equation with nonlocal diffusion. Nonlinearity, 30, (2017), no. 8, 3126-3150.

[13] T. Cazenave and A. Haraux. An introduction to semilinear evolution equations, volume 13 of Oxford Lecture Series in Mathematics and its Applications. The Clarendon Press, Oxford University Press, New York, 1998. Translated from the 1990 French original by Yvan Martel and revised by the authors.

[14] E. Chasseigne, M. Chaves, and J. D. Rossi. Asymptotic behavior for nonlocal diffusion equations. J. Math. Pures Appl. (9), 86, (2006), no. 3, 271-291.

[15] C. Cattaneo. The spectrum of the continuous Laplacian on a graph. Monatsh. Math., 124(3), (1997), 215-235.

[16] C. Cortazar, M. Elgueta, F. Quiros and N. Wolanski. Asymptotic behavior for a nonlocal diffusion equation in domains with holes. Arch. Ration. Mech. Anal. 205, (2012), no. 2, 673-697.

[17] C. Cortazar, M. Elgueta, F. Quiros and N. Wolanski. Asymptotic behavior for a nonlocal diffusion equation in exterior domains: the critical two-dimensional case. J. Math. Anal. Appl. 436, (2016), no. 1, 586-610.

[18] C. Cortazar, M. Elgueta, J. D. Rossi and N. Wolanski. How to approximate the heat equation with Neumann boundary conditions by nonlocal diffusion problems. Arch. Ration. Mech. Anal. 187, (2008), no. 1, 137-156.

[19] E. B. Davies. Heat kernels and spectral theory, volume 92 of Cambridge Tracts in Mathematics. Cambridge University Press, Cambridge, 1990.

[20] Klaus-Jochen Engel and Marjeta Kramar Fijavž. Waves and diffusion on metric graphs with general vertex conditions. Evol. Equ. Control Theory, 8(3):633-661, 2019.

[21] P. Fife. Some nonclassical trends in parabolic and parabolic-like evolutions. Trends in nonlinear analysis, 153-191, Springer, Berlin, 2003.

[22] N. Fournier and P. Laurençot. Local properties of self-similar solutions to Smoluchowski's coagulation equation with sum kernels. J. Funct. Anal., 233, (2006), 351-379.

[23] J. Garcia-Melian and F. Quiros, Fujita exponents for evolution problems with nonlocal diffusion. J. Evol. Equ. 10, (2010), no. 1, 147-161.

[24] G. Gilboa and S. Osher, Nonlocal linear image regularization and supervised segmentation. UCLA CAM Report 06-47, (2006).

[25] S. Haeseler. Heat kernel estimates and related inequalities on metric graphs. Preprint ArXiv 1101.3010. https://arxiv.org/abs/1101.3010. 
[26] L. I. Ignat, T. I. Ignat and D. Stancu-Dumitru. A compactness tool for the analysis of nonlocal evolution equations. SIAM J. Math. Anal. 47, (2015), no. 2, 1330-1354.

[27] L. I. Ignat and J. D. Rossi. A nonlocal convection-diffusion equation. J. Funct. Anal., 251(2), (2007), 399-437.

[28] L. I. Ignat and J. D. Rossi. Refined asymptotic expansions for nonlocal diffusion equations. J. Evol. Equ., 8, (2008), no. 4, 617-629.

[29] L. I. Ignat and J. D. Rossi. Decay estimates for nonlocal problems via energy methods. J. Math. Pures Appl. (9), 92(2), (2009), 163-187.

[30] L. I. Ignat and J. D. Rossi. Asymptotic expansions for nonlocal diffusion equations in $L^{q}$-norms for $1 \leq q \leq 2$. J. Math. Anal. Appl., 362, (2010), no. 1, 190-199.

[31] L. I. Ignat, J. D. Rossi and A. San Antolin, Lower and upper bounds for the first eigenvalue of nonlocal diffusion problems in the whole space. J. Differential Equations, 252, (2012), no. 12, 6429-6447.

[32] S. Kindermann, S. Osher and P. W. Jones. Deblurring and denoising of images by nonlocal functionals. Multiscale Model. Simul., 4, (2005), 1091-1115.

[33] V. Kostrykin and R. Schrader. Laplacians on metric graphs: eigenvalues, resolvents and semigroups. In Quantum graphs and their applications, volume 415 of Contemp. Math., pages 201-225. Amer. Math. Soc., Providence, RI, 2006.

[34] M. Kramar Fijavž, D. Mugnolo and E. Sikolya. Variational and semigroup methods for waves and diffusion in networks. Appl. Math. Optim., 55(2):219-240, 2007.

[35] P. Kuchment. Quantum graphs: an introduction and a brief survey. In Analysis on graphs and its applications, volume 77 of Proc. Sympos. Pure Math., pages 291-312. Amer. Math. Soc., Providence, RI, 2008.

[36] A. Mogilner and L. Edelstein-Keshet. A non-local model for a swarm. J. Math. Biol., 38, (1999), 534-570.

[37] A. Molino and J. D. Rossi. Nonlocal diffusion problems that approximate a parabolic equation with spatial dependence. Z. Angew. Math. Phys. 67, (2016), no. 3, Art. 41, 14 pp.

[38] D. Mugnolo. Gaussian estimates for a heat equation on a network. Netw. Heterog. Media, 2(1), (2007), $55-79$.

[39] El Maati Ouhabaz. Analysis of heat equations on domains, volume 31 of London Mathematical Society Monographs Series. Princeton University Press, Princeton, NJ, 2005.

[40] A. Ponce. An estimate in the spirit of Poincare's inequality. J. Eur. Math. Soc, 6, (2004), no.1, 1-15.

[41] A. Rodriguez-Bernal and S. Sastre-Gomez. Linear non-local diffusion problems in metric measure spaces. Proc. Roy. Soc. Edinburgh Sect. A, 146, (2016), no. 4, 833-863.

[42] J. Simon. Compact sets in the space $L^{p}(0, T ; B)$. Ann. Mat. Pura Appl. (4) 146 (1987), 65-96.

\section{I. IGNAT}

Institute of Mathematics "Simion Stoilow" of the Romanian Academy, Centre Francophone en Mathématique, 21 Calea Grivitei Street, 010702 Bucharest, ROMAniA.

ICUB, The Research Institute of the University of Bucharest, University of Bucharest, 36-46 Bd. M. Kogalniceanu, 050107, Bucharest, ROMAniA.

Email address: liviu.ignat@gmail.com

Web page: http://www.imar.ro/ lignat

J. D. Rossi

Dpto. de Matemáticas, FCEyn, Universidad de Buenos Aires, 1428, Buenos Aires, ARGEntinA.

Email address: jrossi@dm.uba.ar

Web page: http://mate.dm.uba.ar/ jrossi/ 
A. SAn Antolin

Departamento de Análisis Matemático, Universidad de Alicante, Ap. correos 99, 03080, Alicante, SPAIN.

Email address: angel.sanantolin@ua.es 